\title{
THE BALLOON-BORNE LARGE APERTURE SUBMILLIMETER TELESCOPE: BLAST
}

\author{
E. Pascale, ${ }^{1,2}$ P. A. R. Ade, ${ }^{2}$ J. J. Bock, ${ }^{3,4}$ E. L. Chapin, ${ }^{5}$ J. Chung, ${ }^{1,5}$ M. J. Devlin, ${ }^{6}$ S Dicker, ${ }^{6}$ M. Griffin, ${ }^{2}$ \\ J. O. Gundersen, ${ }^{7}$ M. Halpern, ${ }^{5}$ P. C. Hargrave, ${ }^{2}$ D. H. Hughes, ${ }^{8}$ J. Klein, ${ }^{6}$ C. J. MacTavish, ${ }^{1}$ \\ G. Marsden, ${ }^{5}$ P. G. Martin, ${ }^{9,10}$ T. G. Martin, ${ }^{1}$ P. Mauskopf, ${ }^{2}$ C. B. Netterfield, ${ }^{1,10}$ \\ L. Olmi, ${ }^{11,12}$ G. Patanchon, ${ }^{5,13}$ M. Rex, ${ }^{6}$ D. Scott, ${ }^{5}$ C. Semisch, ${ }^{6}$ N. Thomas, ${ }^{7}$ \\ M. D. P. Truch, ${ }^{14}$ C. Tucker, ${ }^{2}$ G. S. Tucker, ${ }^{14}$ \\ M. P. Viero, ${ }^{10}$ AND D. V. Wiebe ${ }^{1}$ \\ Received 2007 May 29; accepted 2008 March 20
}

\begin{abstract}
The Balloon-borne Large Aperture Submillimeter Telescope (BLAST) is a suborbital surveying experiment designed to study the evolutionary history and processes of star formation in local galaxies (including the Milky Way) and galaxies at cosmological distances. The BLAST continuum camera, which consists of 270 detectors distributed between three arrays, observes simultaneously in broadband (30\%) spectral windows at 250,350 , and $500 \mu \mathrm{m}$. The optical design is based on a $2 \mathrm{~m}$ diameter telescope, providing a diffraction-limited resolution of $30^{\prime \prime}$ at $250 \mu \mathrm{m}$. The gondola pointing system enables raster mapping of arbitrary geometry, with a repeatable positional accuracy of $\sim 30^{\prime \prime}$; postflight pointing reconstruction to $\lesssim 5^{\prime \prime} \mathrm{rms}$ is achieved. The onboard telescope control software permits autonomous execution of a preselected set of maps, with the option of manual override. In this paper we describe the primary characteristics and measured in-flight performance of BLAST. BLAST performed a test flight in 2003 and has since made two scientifically productive long-duration balloon flights: a $100 \mathrm{hr}$ flight from ESRANGE (Kiruna), Sweden to Victoria Island, northern Canada in $2005 \mathrm{June}$; and a $250 \mathrm{hr}$, circumpolar flight from McMurdo Station, Antarctica, in 2006 December.
\end{abstract}

Subject headings: balloons — galaxies: evolution — instrumentation: miscellaneous — stars: formation submillimeter

Online material: color figure

\section{INTRODUCTION}

We have built and flown a balloon-borne submillimeter observatory, designed to study the star formation in our Galaxy, in nearby resolved galaxies, and in high-redshift starburst galaxies by observing at several hundred microns, a wavelength range that is not available from the ground.

The Balloon-borne Large Aperture Submillimeter Telescope (BLAST) is a stratospheric balloon-borne $2 \mathrm{~m}$ telescope that observes the sky with bolometric detectors operating in three $30 \%$ wide bands at 250,350, and $500 \mu \mathrm{m}$. The diffraction-limited op-

\footnotetext{
1 Department of Physics, University of Toronto, Toronto, ON M5S 1A7, Canada; enzo@physics.utoronto.ca.

2 Department of Physics and Astronomy, Cardiff University, Cardiff CF24 3AA, UK.

3 Jet Propulsion Laboratory, Pasadena, CA 91109-8099.

4 Observational Cosmology, MS 59-33, California Institute of Technology, Pasadena, CA 91125.

5 Department of Physics and Astronomy, University of British Columbia, Vancouver, BC V6T 1Z1, Canada.

6 Department of Physics and Astronomy, University of Pennsylvania, Philadelphia, PA 19104.

7 Department of Physics, University of Miami, Coral Gables, FL 33146.

8 Instituto Nacional de Astrofísica Óptica y Electrónica (INAOE), 72000 Puebla, Mexico.

9 Canadian Institute for Theoretical Astrophysics, University of Toronto, Toronto, ON M5S 3H8, Canada.

10 Department of Astronomy and Astrophysics, University of Toronto, Toronto, ON M5S 3H4, Canada.

11 Istituto di Radioastronomia, I-50125 Florence, Italy.

12 Physics Department, University of Puerto Rico, Rio Piedras Campus, San Juan, PR 00931.

13 Laboratoire APC, 75205 Paris, France.

14 Department of Physics, Brown University, Providence, RI 02912.
}

tics are designed to provide BLAST with a resolution of $30^{\prime \prime}, 42^{\prime \prime}$, and $60^{\prime \prime}$ at the three wave bands, respectively. The detectors and cold optics are adapted from those to be used on the SPIRE instrument on Herschel (Griffin et al. 2003).

BLAST addresses important Galactic and cosmological questions regarding the formation and evolution of stars, galaxies, and clusters (Devlin et al. 2004) by providing the first large-area $\left(\sim 0.8-200 \mathrm{deg}^{2}\right)$ surveys of unique spectral coverage, angular resolution, and sensitivity.

The primary scientific goals of BLAST are (1) to conduct confusion-limited and shallower wide-area extragalactic surveys to constrain the redshift distribution, star formation history, and evolution of optically obscured luminous galaxies by measuring photometric redshifts (derived from the BLAST colors and complementary data; Hughes et al. 2002); (2) to study the spatial clustering of this population; (3) to improve our understanding of the earliest stages of star formation by determining the physical properties and mass function of cold prestellar cores and the efficiency of star formation within different Galactic environments; and (4) to investigate the nature and structure of the interstellar medium by making the highest resolution maps to date of diffuse Galactic emission at these wavelengths.

The BLAST bands bracket the peak of the thermal radiation emitted by dust at temperatures of around $10 \mathrm{~K}$. Assuming that the temperature range of dust in submillimeter galaxies is $\sim 30$ $60 \mathrm{~K}$, BLAST can explore redshifts from 1 to 5 (Hughes et al. 2002). The primary advantage of BLAST over existing submillimeter bolometer arrays such as SCUBA and SHARC is its enhanced sensitivity at wavelengths $\leq 500 \mu \mathrm{m}$ (where the far-IR background peaks) due to the increased atmospheric transmission at balloon altitudes. With sensitivities of $250 \mathrm{mJy} \mathrm{s}^{1 / 2}$, the 


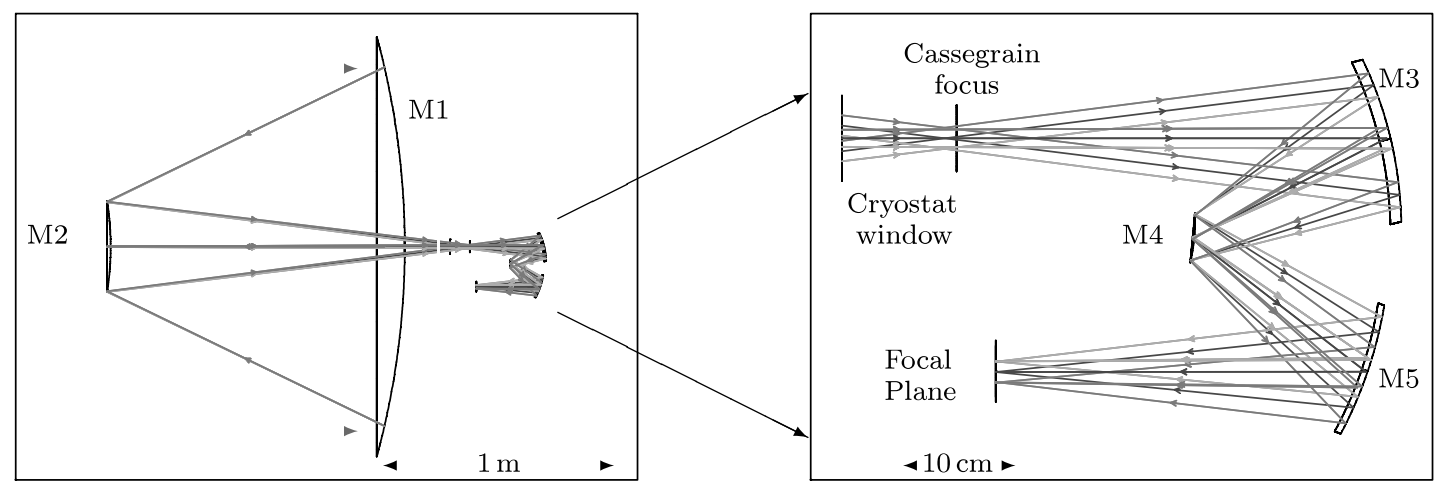

FIG. 1.-Optical layout of the BLAST05 telescope and receiver is shown on the left, and the $1.5 \mathrm{~K}$ optics, located within the cryostat, are shown in expanded view on the right. The image of the sky formed at the input aperture is reimaged onto the bolometer detector array at the focal plane. The mirror M4 serves as a Lyot stop defining the illumination of the primary mirror for each element of the bolometer array. The three wavelength bands are separated by a pair of dichroic beam splitters (not shown), which are arranged in a direction perpendicular to the plane, between M5 and the focal plane. [See the electronic edition of the Journal for a color version of this figure.]

BLAST mapping speed is 10 times faster than the design goal for SCUBA 2 at $500 \mu \mathrm{m}$ (Holland et al. 2006) and more than 100 times the mapping speed of the pioneering flights of the FIR balloonborne telescope PRONAOS (Lamarre et al. 1998). BLAST is complemented at shorter wavelengths by surveys from IRAS, ISO, Spitzer, and Akari, and at longer wavelengths by SCUBA 2, LMT, and ALMA, to constrain spectral energy distributions. Spitzer's higher resolution will also provide accurate astrometry for many of the BLAST sources. BLAST complements the Herschel satellite by testing detectors and filters that are similar to those to be used in the SPIRE instrument (Griffin et al. 2003). Furthermore, the results from BLAST will be available early enough to influence the design (depth and area) of future SPIRE surveys. In addition, BLAST will complement the large-area spectroscopic Galactic surveys of SWAS, provide submillimeter targets for the Fabry-Perot spectrograph SAFIRE on SOFIA, and impact the design of the scientific case for the next generation of submillimeter surveys from space (e.g., SPICA and SAFIR, and later SPIRIT and SPECS).

BLAST has made three flights to date. A $24 \mathrm{hr}$ test flight (BLAST03) from Fort Sumner, New Mexico, in 2003 September demonstrated the performance of the instrument subsystems.

BLAST made its first science flight (BLAST05) in June of 2005 , flying on a $1.1 \times 10^{6} \mathrm{~m}^{3}$ balloon from the Swedish Space Corporation base of ESRANGE, near Kiruna, Sweden, to Victoria Island in northern Canada. During this 4.5 day flight at an average altitude of $38 \mathrm{~km}$, the instrument performed well, except for degraded optical performance. This was possibly due to a failure of structural elements in the carbon fiber mirror. The result was decreased resolution, which had a significant impact on our ability to achieve our extragalactic science goals. BLAST acquired $100 \mathrm{hr}$ of data on Galactic targets, providing some of the first arcminute resolution images at these wavelengths (Chapin et al. 2008). In this flight, surveys of five star-forming regions were conducted, including the well-studied Cygnus X field, three regions of intermediate/high-velocity cirrus, the Cassiopeia A supernova remnant, and several individual bright targets.

BLAST made its second science flight (BLAST06) in 2006 December, flying from the Williams Field Long Duration Balloon (LDB) facility near McMurdo Station in Antarctica. In this flight, the instrument met its performance goals in resolution, sensitivity, and pointing. The flight was terminated after $250 \mathrm{hr}$ of data were acquired. Unfortunately, an anomaly with the parachute separation system prevented the parachute from being released from the gondola after landing. The parachute dragged BLAST for $200 \mathrm{~km}$ before it came to rest in a crevasse field on the Antarctic Plateau. While most of the instrument was destroyed, the hard drives containing the data were found nearby and the complete data set recovered. In this flight, BLAST conducted shallow $\left(10 \mathrm{deg}^{2}\right)$ and deep, confusion-limited $\left(0.8 \mathrm{deg}^{2}\right)$ extragalactic surveys of the Chandra Deep Field-South (CDF-S), as well as a $10 \mathrm{deg}^{2}$ region near the south ecliptic pole. A Galactic survey in Vela mapped 52 and $200 \mathrm{deg}^{2}$ of sky in deep and shallow observations, respectively. Surveys of several Galactic and extragalactic selected targets were also conducted.

The telescope and optics are described in $\S 2$, while $\S \S 3$ and 4 focus on detector arrays and cryogenics. In $\S \S 5-7$ we provide detailed information regarding the gondola, command and control, and pointing systems. In $\S \S 8-11$ we provide overviews of the unique thermal and power requirements and a description of the pointing performance.

\section{TELESCOPE}

The basic BLAST optical configuration is shown in Figure 1. Incoming radiation is collected by the telescope (M1 and $\mathrm{M} 2$ in the figure), which is located on the inner frame of the gondola (described in $\S 5$ ), along with the cryogenic receiver (§ 4). The telescope focus is located about $20 \mathrm{~cm}$ behind the optical surface of the primary. Cold $(1.5 \mathrm{~K})$ reimaging optics (M3, M4, and M5) in the receiver relay the sky image to the detector focal planes. A baffled Lyot stop (M4) is located at the position of an image of the primary mirror. This blocks stray radiation due to scattering and diffraction. After M5 the radiation is divided into three spectral bands through the use of two low-pass edge dichroic filters (Ade et al. 2006). An aperture at the center of the Lyot stop is made to match the one at the center of the primary mirror to reduce loading. This same hole accommodates a commandable thermal source, which provides a stable and repeatable optical signal (calibration lamp) to monitor detector responsivity drifts (Hargrave et al. 2006). The optical design is optimized to deliver a diffractionlimited image of the sky at the detector focal planes, with $60^{\prime \prime}$ and $30^{\prime \prime}$ beams at 500 and $250 \mu \mathrm{m}$, respectively. The telescope designs of BLAST05 and BLAST06 are discussed in this section, and the relevant parameters can be found in Table 1 .

\subsection{Focal Plane}

The illumination of the Lyot stop and the main reflector depends primarily on the properties of the feed horns in the detector array. BLAST uses $2 f \lambda$-spaced, smooth-walled conical feeds that 
TABLE 1

BLAST Optics Design Summary

\begin{tabular}{|c|c|c|}
\hline Element & BLAST05 & BLAST06 \\
\hline Primary mirror diameter $(\mathrm{m})$ & 2 & 1.8 \\
\hline 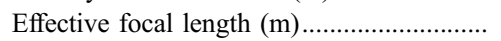 & 10 & 9 \\
\hline Field taper $(\mathrm{dB})$ & -15 & -7.5 \\
\hline 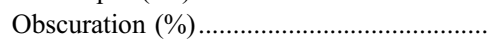 & 14 & 7 \\
\hline FWHM $250 \mu \mathrm{m}(\operatorname{arcsec})$ & 40 & 30 \\
\hline FWHM $350 \mu \mathrm{m}(\operatorname{arcsec})$ & 58 & 42 \\
\hline FWHM $500 \mu \mathrm{m}(\operatorname{arcsec})$ & 75 & 60 \\
\hline
\end{tabular}

NOTES.-The BLAST05 instrument used a larger mirror that was underilluminated. In order to maintain the point-source detectability, the BLAST06 telescope was more aggressively illuminated. The total transmission of the optical system was evaluated with optical CAD software and using the known efficiency of each smooth-walled feed-horn-coupled pixel.

are similar to the SPIRE feeds. Their design and measured characteristics have been described by Chattopadhyay et al. (2003), Rownd et al. (2003), and Griffin et al. (2002). The optical system design is based on the feed-horn nominal $f / 5$ focal ratio and requires the chief rays to be perpendicular to the aperture of the horn, with all the beams overlapping at the Lyot stop. Knowledge of the fundamental propagated electromagnetic mode $\left(\mathrm{TE}_{11}\right)$ is used in a Zemax ${ }^{15}$ physical optics module to verify the final design.

\subsection{BLAST05 Optical Design}

For the 2005 flight, the design incorporated a $2 \mathrm{~m}$ carbon fiber spherical primary mirror with a mass of $32 \mathrm{~kg}$ and a surface rms of $2.4 \mu \mathrm{m}$. It was designed and built by Composite Optics Incorporated ${ }^{16}$ as a technology prototype for the $3.5 \mathrm{~m}$ Herschel telescope. An aluminum correcting secondary mirror, with a diameter of $50 \mathrm{~cm}$, was designed to give a diffraction-limited performance over a $14^{\prime} \times 7^{\prime}$ field of view (FOV) at the telescope focus. The estimated antenna efficiency is $\geq 80 \%$; losses come from a combination of the rms surface roughness of the primary and the quality of the reimaging optics. Two of the reimaging cold elements, M3 and M5, are ellipsoidal mirrors; M2 and the other cold element, M4, compensate for most of the spherical aberration of the primary, whereas M3 and M5 contribute to achieve the correct focal ratio. The perimeter of the primary mirror was structurally weak, forcing M2 to be suspended by a carbon fiber structure attached through the hole in the primary mirror. This geometry resulted in $\sim 12 \%$ blockage of the beam. A detailed discussion about BLAST05 optical design and optimization can be found in Olmi (2001, 2002). The $2 \mathrm{~m}$ primary mirror was underilluminated by setting the entrance pupil diameter to $188 \mathrm{~cm}$, and the reflector was strongly tapered, resulting in a field taper of about $-15 \mathrm{~dB}$. A Strehl ratio $>0.96$ was achieved over the whole focal plane, and far-field beams had a first sidelobe at $-10 \mathrm{~dB}$. A detailed analysis of the relation between Strehl ratio and aperture efficiency, which is more commonly quoted for filled-aperture antennas, can be found in Olmi \& Bolli (2007).

\subsection{BLAST06 Optical Design}

In the 2006 Antarctic flight, BLAST flew a Ritchey-Chrétien telescope, with a $1.8 \mathrm{~m}$ diameter, aluminum primary mirror with a mass of $114 \mathrm{~kg}$. Originally, the mirror was intended to be used only for the test flight in 2003. However, after the destruction of the carbon fiber mirror on landing of BLAST05, the aluminum

\footnotetext{
15 See http://www.zemax.com.

16 Composite Optics Incorporated (COI), 9617 Distribution Avenue, San Diego, CA 92121.
}

mirror was remachined to improve the quality of the surface from $8 \mu \mathrm{m}$ to better than $4 \mu \mathrm{m} \mathrm{rms}$. This machining was performed by the Precision Engineering Group at Lawrence Livermore National Laboratory. A $40 \mathrm{~cm}$ diameter aluminum secondary was suspended by four carbon fiber struts ${ }^{17}$ (with a zero linear thermal expansion coefficient) attached to the perimeter of the primary mirror. This reduced the obscuration to $\sim 7 \%$ compared to the BLAST05 design. Simulations of in-flight detector loading have shown a reduction of $\gtrsim 40 \%$ for this solution, relative to the BLAST05 configuration. In an attempt to further reduce loading, angled reflecting baffles were installed on the inner surface of each strut to deflect most of the obscured beam to the relatively cold sky. The cold reimaging optics form an ideal Offner relay. In this configuration, M3, M4, and M5 are all spherical and share a common center of curvature; M3 and M5 are concave, while M4 is convex. In order to achieve a diffraction-limited beam of $60^{\prime \prime}$ at $500 \mu \mathrm{m}$ and $30^{\prime \prime}$ at $250 \mu \mathrm{m}$ with a smaller diameter primary compared to BLAST05, M1 was more aggressively illuminated. This was made possible by reducing the diameter of the Lyot stop by about $12 \%$, leading to a field taper of $-7.5 \mathrm{~dB}$ on M1 and $-17 \mathrm{~dB}$ sidelobes in the far-field beams.

\subsection{BLAST06 Focusing System}

The relative distance between the primary and the secondary mirrors has a tolerance of one wavelength before significant image degradation is introduced. Thermal modeling indicated that diurnal temperature fluctuations at balloon altitudes of the aluminum M1 and M2 mirrors could have been as large as $10^{\circ} \mathrm{C}$. The required correction in the relative distance between M1 and M2 was calculated to be $50 \mu \mathrm{m}$ per ${ }^{\circ} \mathrm{C}$.

To correct for the thermal motion, we implemented a motorized system for in-flight refocusing of the secondary. M2 was coupled to its mounting structure via 17-7PH stainless steel leaf springs. Three stepper motor actuators ${ }^{18}\left(2.12 \mathrm{~mm} \mathrm{rev}^{-1}\right.$ lead screw) drive the mirror back and forth to correct for the focus position, as well as adding tip/tilt capability to initially align M2 to M1. Accurate positioning was achieved with differential optical encoders ( $\simeq 1$ count $\mu \mathrm{m}^{-1}$ resolution) factory mounted to the motors and three DC Linear Variable Differential Transformers ${ }^{19}$ (LVDTs). This system allowed for $\pm 5 \mathrm{~mm}$ of motion about the nominal telescope focus, which was sufficient for accurate positioning. The flight computer commands each motor individually by communicating with the stepper motor controllers over an RS485 bus.

Preflight focusing of the system was hampered by the fact that water vapor absorption makes ground-based submillimeter observations in the far field of the telescope (about $10 \mathrm{~km}$ ) impossible. To mitigate this, the secondary mirror was physically offset to three positions so that the focal plane was reimaged at 50,100, and $150 \mathrm{~m}$ from the telescope. The focusing system was then used to fine-tune the focus at each position. These results were extrapolated to determine the far-field focus for the flight.

In flight, the focus of the system was verified and had to be manually adjusted by maximizing the response when scanning over bright point sources: a correction of $300 \mu \mathrm{m}$ to the position set at launch was required. Subsequently, the temperatures of the primary and secondary were monitored and the secondary automatically repositioned to account for thermal variations that

\footnotetext{
${ }^{17}$ Innovative Composite Engineering, 139 East Columbia River Way, Bingen, WA 98605.

${ }_{18}$ Ultra Motion, 22355 Route 48, No. 21, Cutchogue, NY 11935.

19 Macro Sensors, 7300 US Route 130 North, Building 22, Pennsauken, NJ $08110 ; 0.6 \mu \mathrm{m}$ or better repeatability.
} 


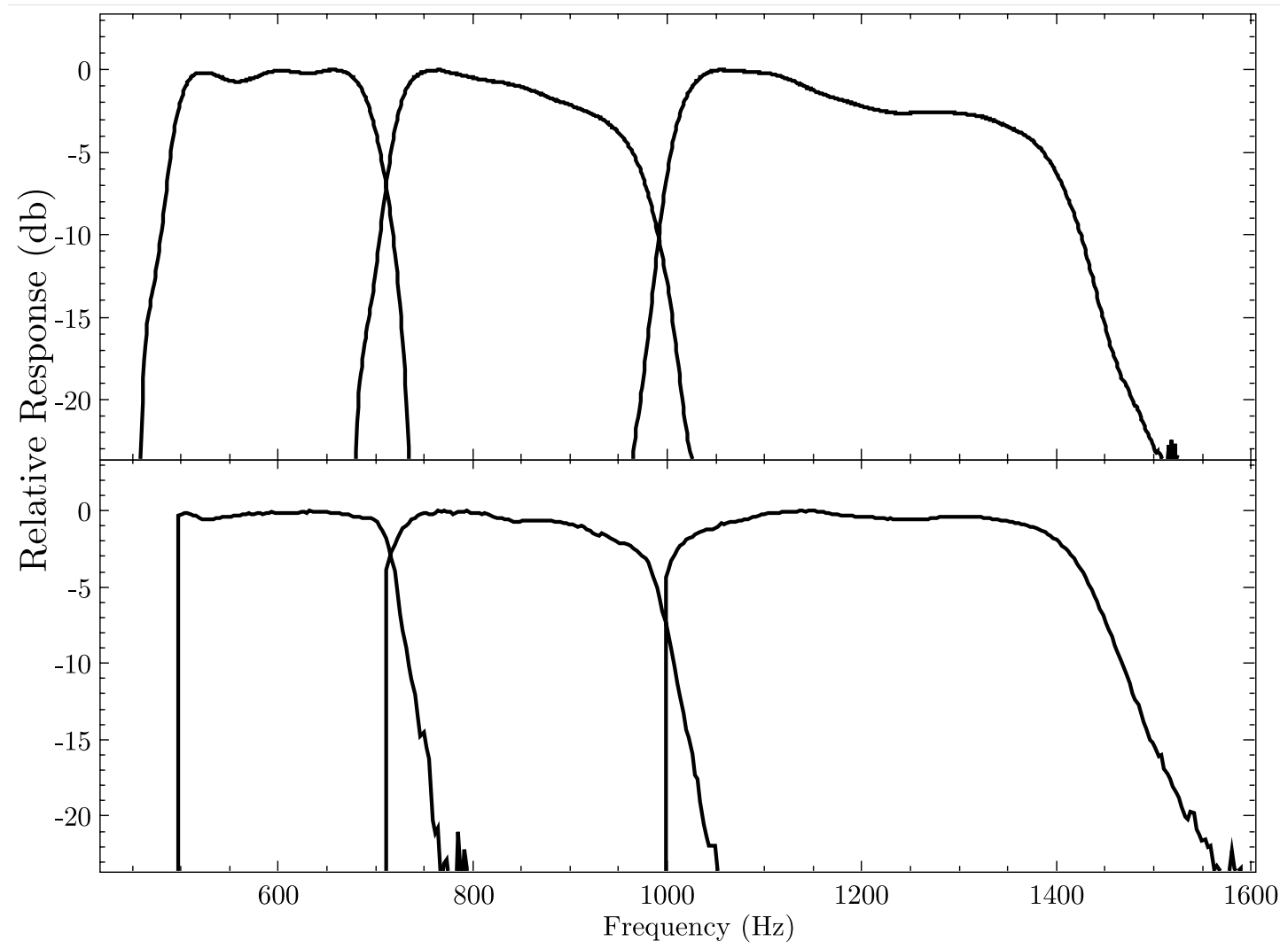

FIG. 2.- Relative spectral response of the three BLAST channels. The BLAST05 bands (top) have been measured with the filter stack in the cryostat. The BLAST06 bands (bottom) are obtained by multiplying the profiles measured for each filter individually; the feed waveguide cutoff is assumed at the longer wavelength. A Fourier transform spectrometer has been used in both cases.

would produce a focus displacement of $100 \mu \mathrm{m}$ or more. The primary and secondary mirror diurnal temperature fluctuations were $\pm 1.5^{\circ} \mathrm{C}$ for most of the flight.

\subsection{Array Bandpass Characteristics}

Low-pass edge dichroic filters (Ade et al. 2006) split the incoming radiation emerging from M5. The first dichroic filter reflects wavelengths shorter than $300 \mu \mathrm{m}$ and transmits longer wavelengths. The bandpass for the $250 \mu \mathrm{m}$ array is further defined by a filter directly in front of the array, which reflects wavelengths shorter than $215 \mu \mathrm{m}$, and by the waveguide cutoff at the exit of each of the feed horns. For the 350 and $500 \mu \mathrm{m}$ arrays, in each case, the band is defined at the short-wavelength end by the dichroic transmission and at the long-wavelength end by the waveguide cutoff. Each band has a 30\% width. The filter stack frequency performance was evaluated with Fourier transform spectroscopy. Bandpasses are shown in Figure 2.

\section{RECEIVER}

The BLAST focal plane consists of arrays of 149,88 , and 43 detectors at 250,350 , and $500 \mu \mathrm{m}$, respectively. The arrays are cooled to a temperature of $270 \mathrm{mK}$. Each array element is a silicon nitride micromesh "spiderweb" bolometer (Bock et al. 1996, 1998). The detector arrays, the feeds, and the mounting scheme are based on the SPIRE instrument design (Turner et al. 2001). Each array hosts a number of diagnostic channels: two dark bolometers (channels that have been capped to avoid illumination), two thermistors, and one resistor. The detector parameters are summarized in Table 2.

The detectors and the readout electronics are designed so that the sensitivity is ultimately limited by the photon "BLIP" noise.
To achieve this performance, other contributions to the bolometer noise have to be controlled. A bolometer is fundamentally limited by phonon noise in the thermal link with the heat sink. The noise equivalent power (NEP; Bock et al. 1996) is NEP = $\gamma\left(4 k_{\mathrm{B}} T^{2} G\right)^{1 / 2}$, where $G$ is the thermal conductance, $T$ is the bath temperature, and $\gamma$ takes into account the properties of the thermal link between the detector and the bath. For a given background load $Q$, the maximum sensitivity is achieved when $G \sim Q / T$ (Mather 1984). The detectors have been optimized for loads of 55, 40, and $30 \mathrm{pW}$ at 250,350 , and $500 \mu \mathrm{m}$, respectively.

TABLE 2

Summary of Relevant Detector Characteristics

\begin{tabular}{|c|c|c|c|}
\hline Element & $250 \mu \mathrm{m}$ & $350 \mu \mathrm{m}$ & $500 \mu \mathrm{m}$ \\
\hline Light detectors ................. & 139 & 88 & 43 \\
\hline Dark pixels ........................ & 2 & 2 & 2 \\
\hline 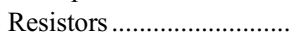 & 1 & 1 & 1 \\
\hline 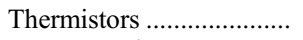 & 2 & 2 & 2 \\
\hline$G\left(\mathrm{pW} \mathrm{K}^{-1}\right) \ldots \ldots \ldots \ldots \ldots \ldots$ & 880 & 640 & 480 \\
\hline$\Delta(K)$ & 50 & 50 & 50 \\
\hline 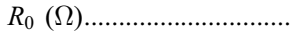 & 55 & 55 & 55 \\
\hline$R_{L}(\mathrm{M} \Omega) \ldots \ldots \ldots \ldots \ldots \ldots \ldots \ldots \ldots \ldots \ldots \ldots$ & $7+7$ & $7+7$ & $7+7$ \\
\hline$\tau(\mathrm{ms})$ & 2 & 2 & 2 \\
\hline Temperature $(\mathrm{mK}) . . . \ldots \ldots . .$. & 270 & 270 & 270 \\
\hline $\mathrm{NEP}\left(\mathrm{W} \mathrm{Hz}^{-1 / 2}\right) \ldots \ldots \ldots \ldots$ & $3 \times 10^{-17}$ & $3 \times 10^{-17}$ & $3 \times 10^{-17}$ \\
\hline
\end{tabular}

NoTEs.-Bolometer parameters are given for a typical array pixel. Parameters $\Delta$ and $R_{0}$ define the bolometric model, $R(T)=R_{0} e^{(\Delta / T)^{1 / 2}}$, while the optical time constant, $\tau$, and the thermal conductance, $G$, define its thermal behavior. The NEP is computed at $1 \mathrm{~Hz}$. 


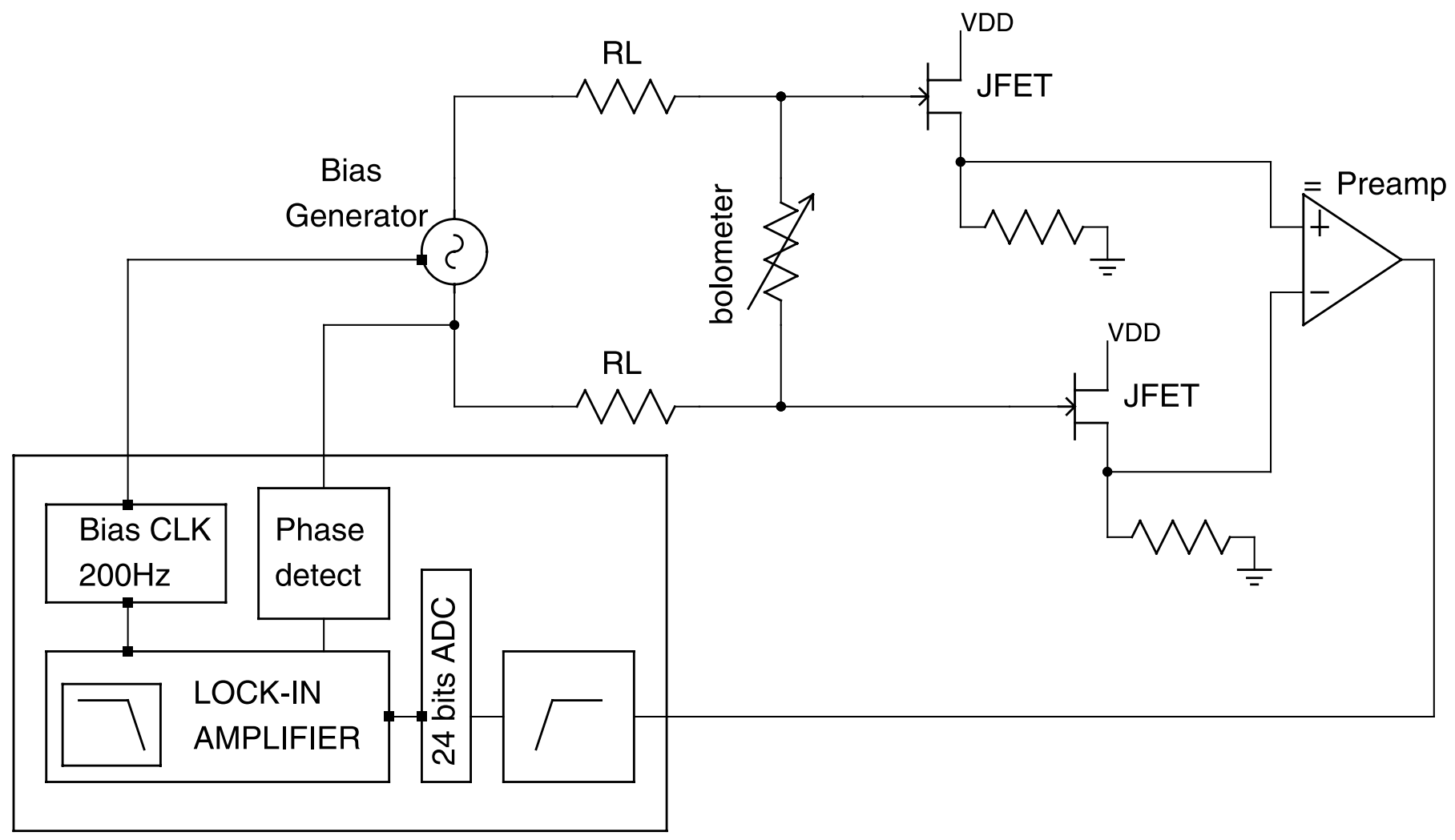

FIG. 3.-Schematic diagram of the detector readout electronics. A 200.32 Hz sine wave biases each bolometer across two $7 \mathrm{M} \Omega$ load resistors (RL). JFET pairs buffer the detector signal to the warm electronics. The two signals are then differenced and digitized. A digital lock-in rectifies and filters the output, sending it to the flight computers over the BLASTbus.

The detector transfer function is measured using cosmic-ray hits, as described in Crill et al. (2003). We have found typical time constants of $2 \mathrm{~ms}$ with a dispersion of $15 \%$ across the arrays.

An additional advantage to the spiderweb bolometer is the relatively small cross section to cosmic rays. Balloon flights at high latitudes have about 100 times the midlatitude cosmic-ray flux. The detected cosmic-ray flux was $1.5,2$, and 6 events per bolometer per minute during both science flights at 250,350, and $500 \mu \mathrm{m}$, respectively.

\subsection{Readout Electronics}

A schematic diagram of the readout electronics is shown in Figure 3. Each bolometer is preamplified with a Siliconix U401 differential JFET with 5-7 nV Hz ${ }^{-1 / 2}$ noise at $\nu>100 \mathrm{~Hz}$. Compact 24 channel JFET modules are integrated into the design of the cryostat, allowing us to sink their power dissipation $(240 \mu \mathrm{W}$ per pair) to the vapor-cooled shield (see $\S 4$ ) and decrease the load on the helium bath by approximately $60 \mathrm{~mW}$. The JFETs are operated at a temperature of $\sim 145 \mathrm{~K}$. Their output is then amplified using the Analog AD624 instrumentation amplifier $\left(5 \mathrm{nV} \mathrm{Hz}^{-1 / 2}\right)$ with a $100 \mathrm{~Hz}$ wide bandpass filter, centered at $208 \mathrm{~Hz}$. To mitigate the effects of $1 / f$ noise, the detectors are $\mathrm{AC}$ biased with a sine wave at $200.32 \mathrm{~Hz}$. A reference square wave at this frequency is generated by dividing down the main $32 \mathrm{MHz}$ clock serving the readout electronics, making the bias synchronous with the sampling. This signal is then split into three paths (one per band), stabilized to a voltage amplitude that is selectable from 0 to $300 \mathrm{mV}$ with a resolution of 7 bits, and filtered into a sine wave using lownoise operational amplifiers (Analog OP470). The bias is delivered to the detectors and a reference is sent back to the Data Acquisition System (DAS) where a digital lock-in is implemented on the DSP (Analog ADSP21062) in the readout electronics. The bolometric $\mathrm{AC}$ signals and the bias reference are digitized with a 24 bit $\Sigma \Delta$ (Burr-Brown ADS1252) fed by a low-noise dual instrumental amplifier (Burr-Brown INA2128) and sampled at $10 \mathrm{kHz}$. The reference is phase locked and a numerical sine wave is generated in-phase at the bias frequency (the clock is shared). The rectified signal is then low-passed with a four-stage boxcar filter having a first null at $50.08 \mathrm{~Hz}$ and decimated to match the DAS sampling frequency $(100.16 \mathrm{~Hz})$.

The resulting flat-phase filter is well approximated by a Gaussian and is computationally efficient in the time domain, allowing up to 25 independent channels to be locked in on a single DSP. Only the real part of the signal is sampled, hence the relative phase between signal and reference must be set manually. Since the phase difference is a function of the bolometer impedance, it needs to be adjusted in flight along with bias levels. This is achieved by maximizing the signal from the pulsed calibration lamp at the Lyot stop.

The overall data acquisition electronics noise is kept below the estimated photon noise and provides stability to low frequencies $(<30 \mathrm{mHz})$, which allows the sky to be observed in a slowly scanned mode.

\subsection{Detector Noise}

The instrument is designed to be limited by photon noise. The error budget, which drove the telescope and readout electronics design, accounted for (1) the photon noise arising from astrophysical backgrounds and emissivity of the optical surfaces, (2) the bolometer noise budget, and (3) the readout noise. A noise equivalent flux density (NEFD) of $250 \mathrm{mJy} \mathrm{s}^{1 / 2}$ was expected from this analysis. 


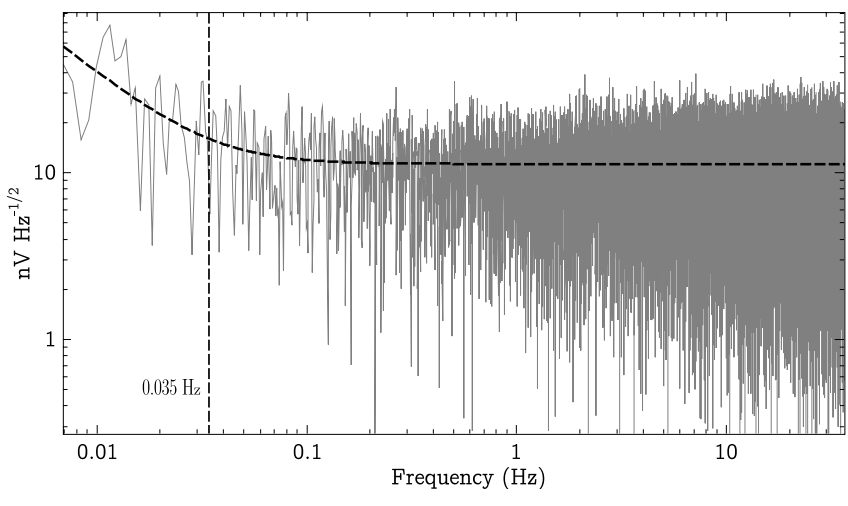

FIG. 4.-A $250 \mu \mathrm{m}$ detector noise PSD. The time stream was acquired during the BLAST05 flight and analyzed as explained in Patanchon et al. (2008): (1) the time stream has been deglitched, (2) the detector and electronics transfer functions have been deconvolved from the data, and (3) a common mode signal, synchronous to each channel in an array, has been removed in the time domain. The PSD is shown before any gain. The dashed line is a brown-noise fit to the PSD, which shows a white-noise level at $11 \mathrm{nV} \mathrm{Hz}^{-1 / 2}$ and a $1 / f$ "knee" at $35 \mathrm{mHz}$ (indicated by the vertical dashed line).

The voltage noise is determined by taking the power spectral density (PSD) of the time stream acquired in flight, and it is shown in Figure 4 for a representative $250 \mu \mathrm{m}$ detector during the BLAST05 flight. Before taking the PSD, each time stream is processed as described in Truch et al. (2008) and Patanchon et al. (2008), which involves deglitching, deconvolution of the bolometer and electronics transfer functions, and removal of common mode signals, synchronous to each detector in an array.

The voltage noise is well described by a brown-noise model. The white-noise level is $11 \mathrm{nV} \mathrm{Hz}^{-1 / 2}$, and the $1 / f$ "knee" is at $\sim 35 \mathrm{mHz}$. Using the responsivities in Truch et al. (2008), we obtain a noise of $\sim 15 \mathrm{MJy} \mathrm{sr}^{-1} \mathrm{~Hz}^{-1 / 2}$. A similar analysis at the longer wavelengths gives $\sim 7$ and $4 \mathrm{MJy} \mathrm{sr}^{-1} \mathrm{~Hz}^{-1 / 2}$ at 350 and $500 \mu \mathrm{m}$, respectively. Within a single array the pixel noise varies by $20 \%$.

Measured noise levels are consistent with the design target for the NEFD for nominal beam sizes.

\section{CRYOGENICS}

The cryostat houses most of the receiver system (Fig. 5) and was fabricated by Precision Cryogenics. ${ }^{20}$ It is constructed of aluminum and G10 woven fiberglass reinforced resin epoxy and is maintained at vacuum to prevent thermal convection. Each thermal stage is light tight except the optical path, which is thermally protected with infrared blocking filters (Truch 2007).

Liquid baths of nitrogen and helium maintain the temperatures of the 77 and $4.2 \mathrm{~K}$ stages. In flight, an absolute pressure regulator $^{21}$ is used to maintain approximately $1 \mathrm{~atm}$ above each bath. A vapor-cooled shield (VCS) is located between the nitrogen and helium stages, which is cooled by boil-off gas from the helium bath and reduces the thermal loading on the helium stage. Radiative and conductive loading is $7.2 \mathrm{~W}$ on the nitrogen tank and $71 \mathrm{~mW}$ on the helium tank. An additional effective load of 9 and $7 \mathrm{~mW}$ on the helium tank is due to the liquid drawn into the pumped pot by the capillary (discussed below) and heating required to cycle the ${ }^{3} \mathrm{He}$ refrigerator, respectively. The cryostat holds 43 liters of nitrogen and 32 liters of helium and has an 11.5 day hold time.

\footnotetext{
20 See http://www.precisioncryo.com.

21 Tavco, Inc., 20500 Prairie Street, Chatsworth, CA.
}

A liquid He pumped pot maintains the optics box at $1.5 \mathrm{~K}$. A long, thin capillary connects the helium bath to the pumped pot with a volume of $\sim 100$ milliliters. A pump line between the pot and the exterior of the cryostat keeps the pot at near vacuum, maintaining the temperature at $1.5 \mathrm{~K}$. The pressure difference between the helium bath and the pot forces helium into the pot. On the ground, a vacuum pump is used to evacuate the pump line; at float altitude, the pump line is open to the atmosphere, which is at a pressure $<0.01$ atm.

The bolometers and feed horns are maintained at $<300 \mathrm{mK}$ by the closed-cycle ${ }^{3} \mathrm{He}$ refrigerator. The ${ }^{3} \mathrm{He}$ is condensed by the $1.5 \mathrm{~K}$ pumped pot and collects in the ${ }^{3} \mathrm{He}$ cold stage. The ${ }^{3} \mathrm{He}$ refrigerator is able to extract $5 \mathrm{~J}$ of energy and needs to be recycled every 48-60 hr. The recycling process takes less than $2.5 \mathrm{hr}$.

The cryostat performed as expected during both science flights. The nitrogen and helium baths maintained constant temperature, and the VCS fluctuated by less than $3 \mathrm{~K}$ due to the ${ }^{3} \mathrm{He}$ refrigerator cycles. The pumped pot maintained a temperature below $1.8 \mathrm{~K}$ with fluctuations less than $10 \mathrm{mK}$. During ${ }^{3} \mathrm{He}$ refrigerator cycles the pumped-pot temperature rose as high as $2.5 \mathrm{~K}$. The bolometer temperature was maintained below $300 \mathrm{mK}$ with less than $1 \mathrm{mK}$ fluctuations on hour timescales. The first science flight was too short to test hold time. In the second 12.5 day flight, the helium tank was exhausted 11.5 days after reaching float altitude. The nitrogen tank was not exhausted before termination.

\section{GONDOLA}

The BLAST gondola provides a pointed platform for the telescope and the attachment point to the balloon flight train. The gondola was designed and built by Empire Dynamic Structures, ${ }^{22}$ based on initial designs from members of the BLAST team. Schematics of the gondola are shown in Figures 6 and 7. The breakdown of the mass of various components is given in Table 3.

\subsection{Requirements}

The gondola design is driven by the pointing requirements of the science case. The elevation range of the inner frame, including the $2 \mathrm{~m}$ primary mirror and the $\sim 200 \mathrm{~kg}$ cryostat, is $25^{\circ}-60^{\circ}$. The entire gondola can rotate to any azimuth angle. The in-flight pointing is accurate to $\sim 30^{\prime \prime}$, and postflight pointing reconstruction is accurate to better than $5^{\prime \prime}$. The telescope's primary observing mode is to scan in azimuth at $\sim 0.1^{\circ} \mathrm{s}^{-1}$. All of the mirrors are shaded from solar radiation.

As a consequence of the pointing requirements, the feedback rate of the control system is $\sim 10 \mathrm{~Hz}$. To accommodate this, the gondola is designed to be rigid, with a minimum resonance frequency greater than $14.4 \mathrm{~Hz}$. All mechanical tolerances are set to minimize backlash. All bearings are low friction, low stiction, and treated with low-temperature grease (see $\S$ 5.2.2). The center of mass of the inner frame is on the rotational axis (the elevation axis) so that translations of the gondola (by wind or from the balloon) do not generate torques that reorient the telescope.

A target weight of $\sim 2000 \mathrm{~kg}$ was set for the entire system, and the moment of inertia was estimated to be $\sim 4500 \mathrm{~kg} \mathrm{~m}^{2}$. The system has been engineered to meet the flight criteria set by the Columbia Scientific Balloon Facility (CSBF).

\subsection{Layout}

The gondola frame consists of three components: an outer frame, suspended from the balloon flight train by cables and a pivot motor; an inner frame, which is attached to the outer frame at

22 See http://www.empireds.com. 


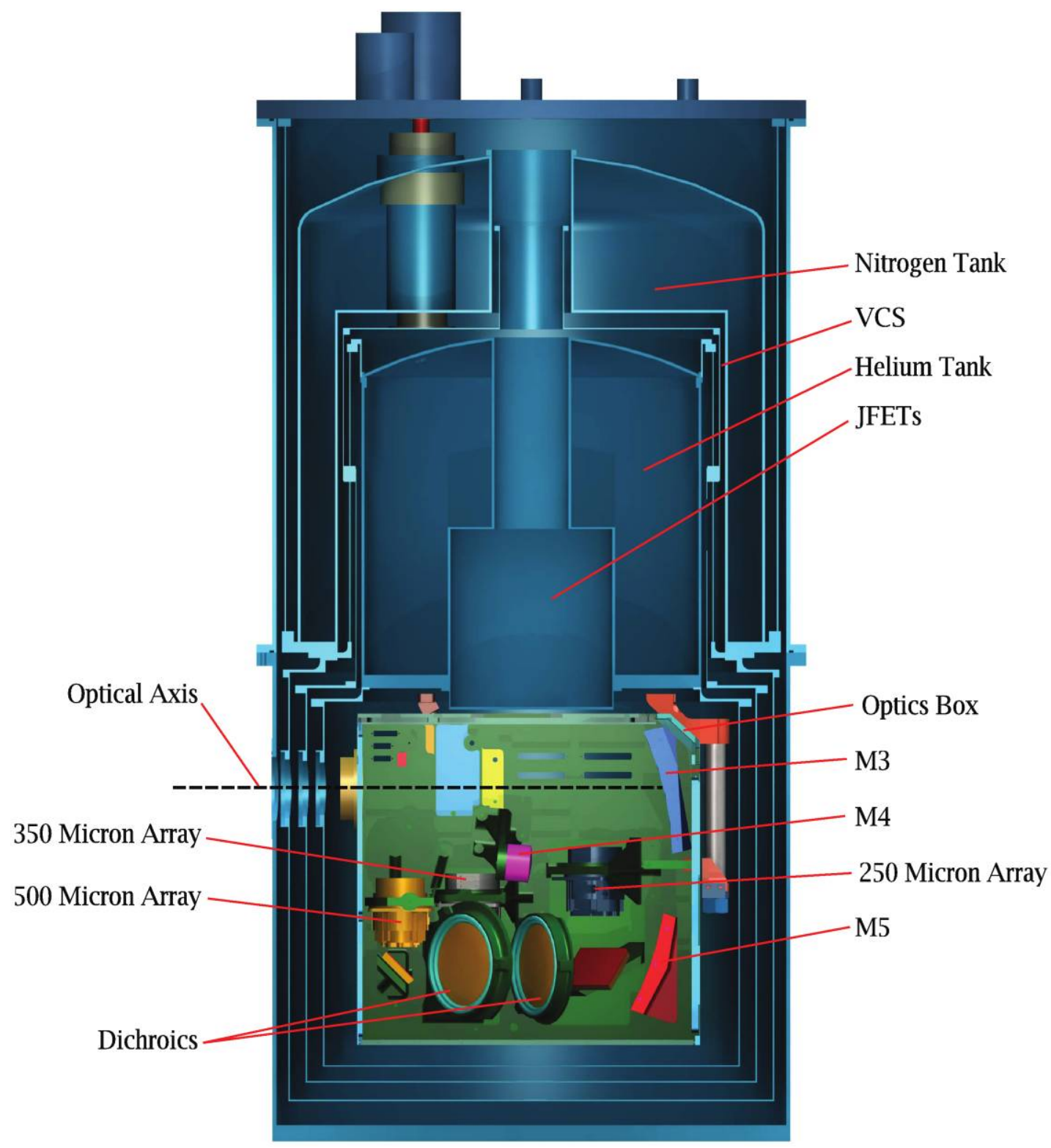

Fig. 5.-Cutaway model of the BLAST receiver showing the optics box. The ${ }^{3} \mathrm{He}$ refrigerator is omitted for clarity. The cryostat is held to the telescope structure via bolts in the top flange and jack screws around the perimeter near the base.

two points along a horizontal axis; and a set of Sun shields that attach to the outer frame. The frame is constructed of lightweight aluminum tubing and I-beam. BLAST incorporates large-diameter torque motors for all motion control.

\subsubsection{Major Components}

The outer frame consists of a horizontal surface, two yokes to support the inner frame, and four legs. The outer frame is pointed in azimuth using a reaction wheel and pivot. It also provides mounts for various electronics boxes, including the flight computers, the CSBF electronics, various pointing sensors, and flight batteries.

The inner frame is made from thin-wall aluminum box beam. It supports the telescope, cryostat, detector readout electronics, gyroscopes, and star cameras. It is attached to the outer frame at two points, defining an axis of rotation.

The back, sides, and bottom of the gondola are surrounded by Sun shields, allowing the telescope to point as low as $25^{\circ}$ in ele- vation. A lightweight aluminum frame encloses the telescope and is covered with extruded polystyrene panels wrapped in aluminized Mylar. The original design shielded the secondary mirror from the Sun at $30^{\circ}$ above the horizon, the highest the Sun rises during an Antarctic flight, with the telescope pointing directly away from the Sun. Additional panels were added for the BLAST06 flight to improve the shading when the telescope is not pointed anti-Sun. The telescope is also protected from solar radiation scattered from the ground. As well as the opening for the telescope, there are forward-facing openings in the Sun shields to allow for cooling of the electronics by radiation (see Fig. 7).

\subsubsection{Attitude Control}

The telescope's attitude is controlled by three torque motors: the reaction wheel, pivot, and elevation motors. The motors are rare earth, permanent-magnet, direct-drive DC torque motors ${ }^{23}$

\footnotetext{
${ }^{23}$ Kollmorgen QT-6205.
} 


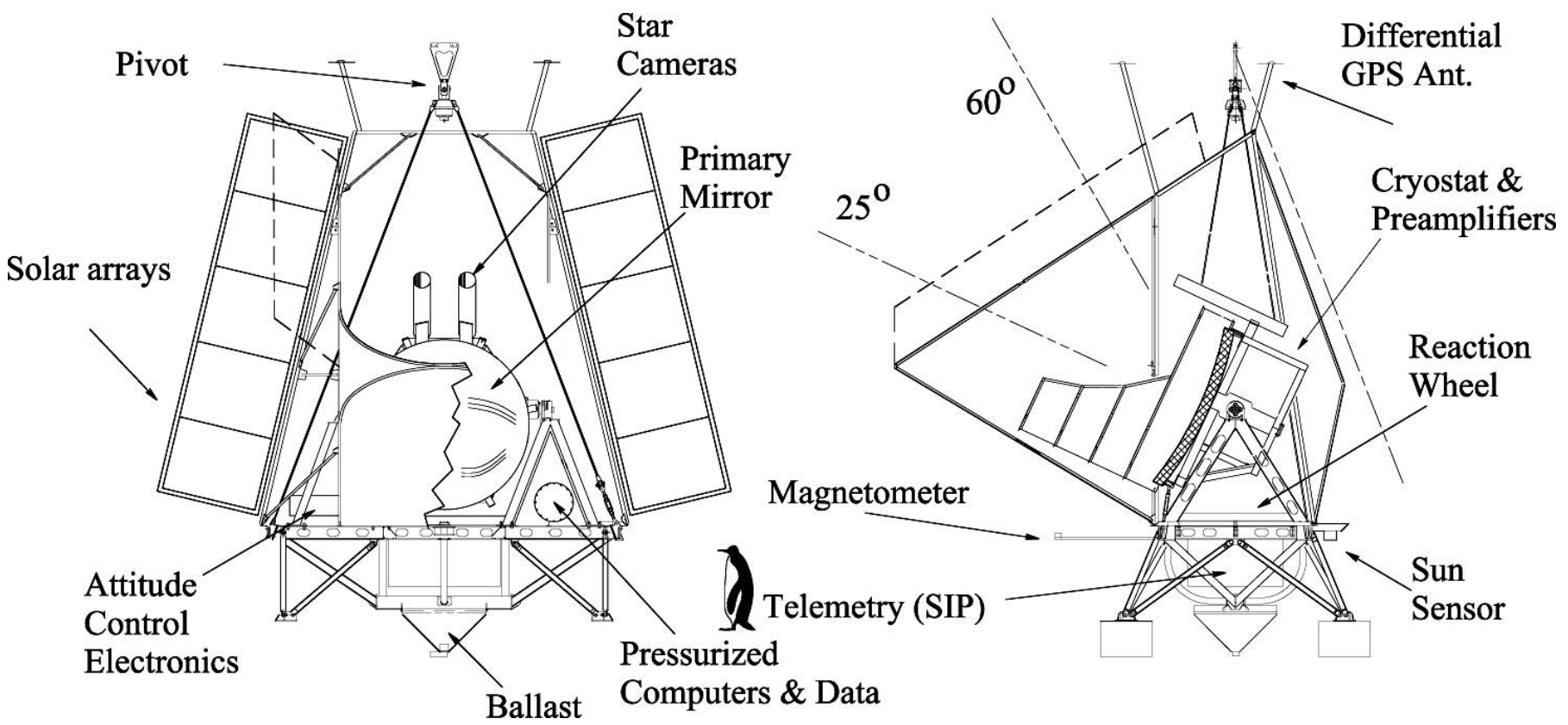

FIg. 6.-Front and side schematic drawings of the BLAST gondola. A $1 \mathrm{~m}$ tall Emperor penguin is shown for scale. The height of the pivot is set by the size of the launch vehicle. The width of the gondola without deploying the solar panel arrays matches the width of the laboratory doors. The inner frame, which can be pointed in elevation, consists of the two star cameras, the telescope and its light baffle, the receiver cryostat, and associated electronics. The telescope baffle shown at right, which was used in 2005, was replaced by a smaller system in 2006. The CSBF solar panels and transmitting antennae are suspended below the structure shown here. The lines marked $25^{\circ}$ and $60^{\circ}$ show the useful range of orientation of the optic axis. The dot-dashed line at the right originating above the pivot shows a $20^{\circ}$ avoidance zone required to avoid accidental contact at launch. The dashed parallelogram at the top in the right panel and to the left in the left panel shows the shape of an extension to the Sun shields added for the Antarctic flight to allow observations to be made further from the anti-Sun direction.

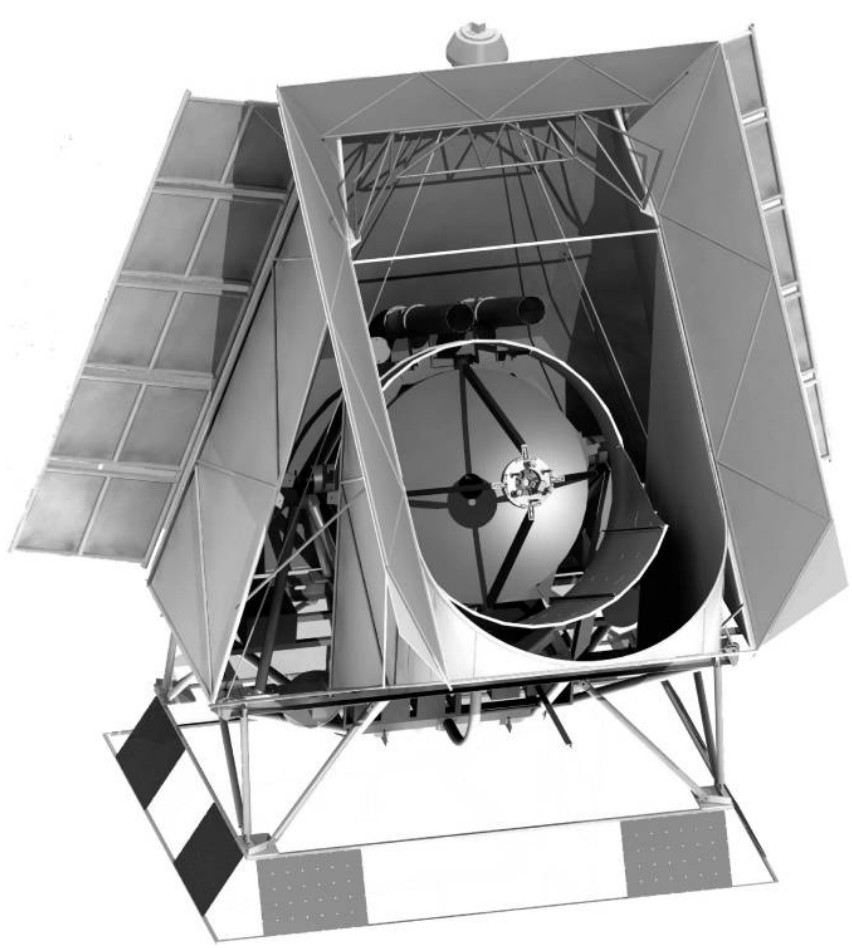

FIG. 7.-CAD model of the gondola, showing BLAST fully assembled in the BLAST06 configuration. The electronics can cool radiatively through the triangular gaps, which are visible in the Sun shields. These gaps always point away from the Sun in flight. with a peak torque of $13.6 \mathrm{~N} \mathrm{~m}$. They are controlled by $50 \mathrm{~A}$ pulse-width modulated servo amplifiers. ${ }^{24}$ The housings, made of hardened steel, are custom designed and incorporate the bearings. The original design called for a dry Teflon bearing lubricant, but we found that low-temperature bearing grease ${ }^{25}$ provides lower friction and stiction.

24 Advanced Motion Controls, model 12A8K.

25 Dow Corning Molykoteß 33.

TABLE 3

Weights of the Main Components on the Payload

\begin{tabular}{|c|c|}
\hline Component & $\begin{array}{c}\text { Weight } \\
(\mathrm{kg})\end{array}$ \\
\hline Inner frame..... & 705 \\
\hline Frame & 120 \\
\hline 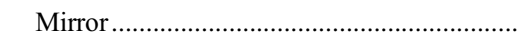 & 115 \\
\hline Cryostat (empty) & 215 \\
\hline Electronics .......................... & 65 \\
\hline Cryogens ......... & 40 \\
\hline Outer frame ....................... & 1100 \\
\hline Batteries & 80 \\
\hline Solar panels & 30 \\
\hline 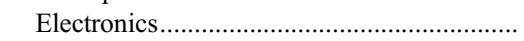 & 110 \\
\hline 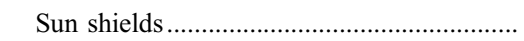 & 195 \\
\hline Frame & 195 \\
\hline 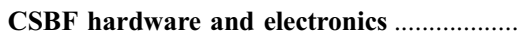 & 215 \\
\hline Total weight at launch & 2020 \\
\hline
\end{tabular}

Notes.-Boldface values refer to the BLAST06 configuration and include the items shown here, as well as all the other elements on the gondola. The total weight at launch was measured at the hook of the launch vehicle before takeoff, and the moment of inertia was $\sim 4500 \mathrm{~kg} \mathrm{~m}^{2}$. 

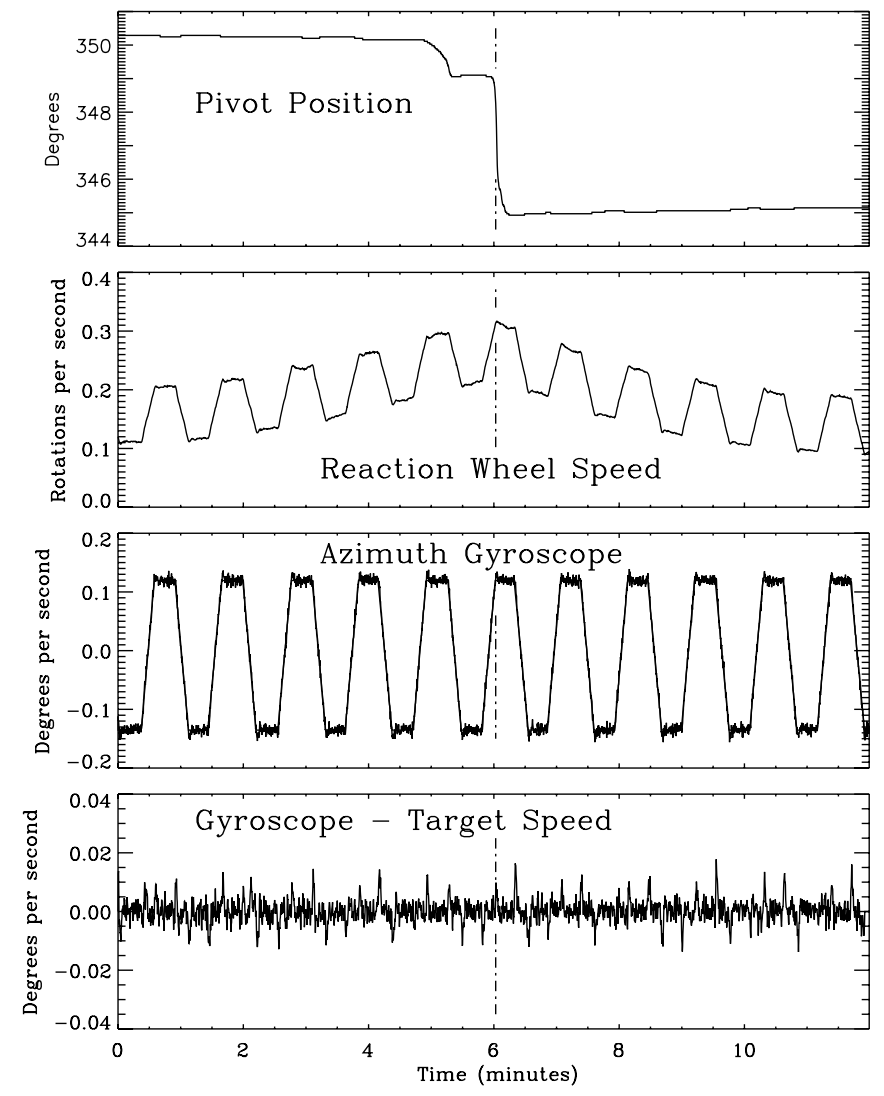

FIg. 8.-Isolation of vertical torques. All panels show data from the same 12 minute segment of the 2006 flight, when the telescope was executing a routine azimuthal scan. The third panel from the top shows a trapezoidal angular velocity profile corresponding to constant angular velocity scans connected by constant angular accelerations. This panel also includes a plot of the desired angular speeds, completely buried by the measured signal. The second panel from the top shows that azimuthal speed is governed by accelerating and decelerating the speed of the reaction wheel. A torque at the pivot proportional to reaction wheel speed reaches threshold and overcomes the stiction in the pivot when the reaction wheel speed is near 0.3 revolutions per second. One such event is marked by the vertical dotdashed line in all panels. The bottom panel contains a plot of the difference between measured gyroscope signal and desired azimuthal speed. Notice that there are small spikes visible corresponding to the corners in the scan speed but that there is no azimuthal motion of the telescope itself coincident with the release of stiction in the pivot.

The telescope is controlled in azimuth by a $1.5 \mathrm{~m}$ reaction wheel, made of $7.6 \mathrm{~cm}$ thick aluminum honeycomb and $480.9 \mathrm{~kg}$ brass disks mounted around the perimeter to maximize the ratio of moment of inertia to mass. The reaction wheel is mounted at the center of the outer frame with its rotation axis going through the pivot. Torquing the gondola against the reaction wheel controls azimuth pointing.

The gondola is subject to external torque from wind shear acting on the gondola and from balloon rotation acting through the flight train. The second term is minimized, but not entirely eliminated, by a vertical axis pivot at the attachment of the gondola to the flight train (see Fig. 6). Since the flywheel motor saturates at $\sim 50 \mathrm{rpm}$, the additional torque motor at the pivot is needed to reduce excess angular momentum, keeping the rotation of the reaction wheel within reasonable limits. Figure 8 demonstrates that this nested servo system, where the reaction wheel is driven to maintain telescope orientation and the pivot is driven to maintain low reaction wheel speed, successfully decouples the telescope from external torques.

The elevation of the inner frame is controlled by a motor mounted on one side of the inner frame at the attachment point to the outer frame. A free bearing provides the connection point on the other side. The motor housing incorporates a 16 bit shaft encoder ${ }^{26}$ which measures the orientation of the inner frame relative to the outer frame. A fluid transfer system, consisting of a pump and two tanks containing $\sim 10$ liters of a high-density nontoxic fluid, ${ }^{27}$ corrects for long-timescale balance offsets induced by the boil-off of the cryogens and the redistribution of cryogens due to changes in elevation.

The balloon environment introduces small oscillations with a period of approximately $25 \mathrm{~s}$ in the pitch and roll of the gondola. Typical amplitudes of $\sim 2^{\prime}$ are observed. A roll damping system consisting of a motor and a small $30 \mathrm{~cm}$ diameter reaction wheel is mounted perpendicular to the flywheel on the outer frame, with the goal of dissipating roll modes. The reaction wheel is made of aluminum and weighs $\sim 2 \mathrm{~kg}$, most of which is at the perimeter. Oscillations in pitch are removed by controlling the elevation of the inner frame. The oscillations in pitch and roll were measured to be $50 \%$ smaller compared to a case when both controllers were off.

\section{COMMAND AND CONTROL}

BLAST is designed to operate autonomously without need of ground commanding. Telescope control is provided by a pair of redundant flight computers with Intel Celeron processors at 366 and $850 \mathrm{MHz}$, running Slackware ${ }^{28}$ Linux 9.2 with Kernel version 2.6.8. The computers are kept at near atmospheric pressure to allow the hard drives to function properly and to provide the appropriate thermal environment for the CPUs.

The flight computers run a single, monolithic program, the "master control program" (mcp), written in C, which performs primary control of all aspects of the telescope, including in-flight pointing solution, motion, commanding, telemetry, data archiving, and thermal control.

The communication link between the telescope and the ground is provided by CSBF through a number of line-of-sight (LOS) transmitters and satellite links. The ground station computers interface with CSBF's ground station equipment to send commands to the payload and display downlinked data. The ground station software uses $\mathrm{kst}^{29}$ and other Linux applications developed by BLAST team members.

\subsection{Gondola Electronics}

The flight computers are built on a passive PCI-bus backplane and contain a single board computer, a four port 8250 serial port extender card, and a custom-made PCI controller card, which interfaces major components as well as the LOS data transmitter. This card interfaces with the BLASTbus, a proprietary RS485 bus with three differential pairs (data, clock, strobe). The BLASTbus protocol is a poll and response architecture composed of a 32 bit request followed by a 32 bit response containing 16 bits of data. The BLASTbus clock runs at $4 \mathrm{MHz}$, giving an effective bandwidth on the BLASTbus of $1 \mathrm{Mbit} \mathrm{s}^{-1}$.

The BLASTbus connects the flight computers to the Attitude Control System (ACS) and DAS. Each DSP card in these systems monitors the BLASTbus. They provide data on request and receive command input from the flight computers via the BLASTbus.

Data are marshaled on the BLASTbus into $100 \mathrm{~Hz}$ frames. Bolometers are polled once per frame, as are the gyroscopes and

\footnotetext{
26 Gurley Precision Instruments A25SB.

27 Dynalene HC-40.

28 See http://www.slackware.com.

29 See http://kst.kde.org.
} 

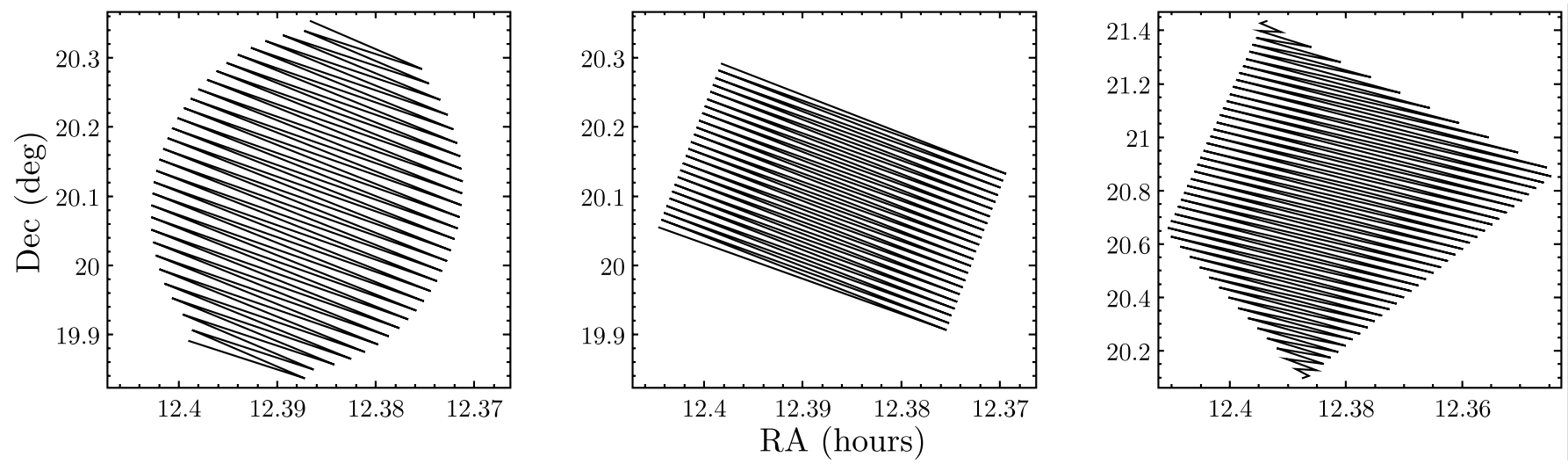

FIG. 9.-Idealized representations of BLAST's three scan modes: from left to right a "cap," a "box," and a "quad." In practice, the gyroscopes are used to control the speed and orientation of each single scan, while star camera-based orientation solutions at the endpoints are used to prevent gyroscope errors from accumulating.

other high-precision pointing data. The majority of housekeeping signals do not need to be polled at $100 \mathrm{~Hz}$; these "slow data" signals are polled at $5 \mathrm{~Hz}$ with groups of 20 multiplexed into a single $100 \mathrm{~Hz}$ channel. The $100 \mathrm{~Hz}$ frames provide the basic data structure for data archival. The frames are written to disk and transmitted by the LOS $1 \mathrm{Mbit} \mathrm{s}^{-1}$ transmitter.

In addition to the BLASTbus, the flight computers also collect data via 8250-style serial connections to the differential GPS, the SIP computers, the lock motor, and the secondary actuators. Ethernet provides further connectivity to the star camera computers and Sun sensor computer. In order to provide data synchronization, the auxiliary channels are written back to the BLASTbus and the star cameras are triggered via the BLASTbus.

\subsection{Pointing Control}

BLAST is a scanning experiment. The primary scan mode involves an azimuthal raster coupled with a slow elevation drift or discrete elevation steps.

Detector response times, $\tau$, together with $1 / f$ noise and star camera integration times set limits on the azimuthal scan rate,

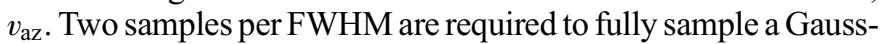
ian beam. The maximum angular rate allowed by the detector $\tau$ is therefore

$$
v_{\mathrm{az}}=\frac{\mathrm{FWHM} / 2}{2 \pi \tau},
$$

or about $0.3^{\circ} \mathrm{s}^{-1}$ for the $30^{\prime \prime}$ beam at $250 \mu \mathrm{m}$ and $\tau \sim 2 \mathrm{~ms}$. Midscan smearing in the star cameras also limits azimuthal scan speeds to about $0.1^{\circ} \mathrm{s}^{-1}$. A scan rate larger than this is acceptable only for relatively small scans where star camera solutions at the turnaround are more frequent. Low-frequency $(1 / f)$ noise sets the largest angular mode that can be constrained to $v_{\mathrm{az}} / f_{0} \lesssim 3^{\circ}$ at $0.1^{\circ} \mathrm{s}^{-1}\left(f_{0}\right.$ is the $1 / f$ knee at $\left.35 \mathrm{mHz}\right)$. Therefore, BLAST scans most of the time at $v_{\mathrm{az}} \simeq 0.1^{\circ} \mathrm{s}^{-1}$, occasionally going twice as fast on small $\left(\lesssim 0.3^{\circ}\right.$ wide $)$ maps. Azimuthal acceleration is limited to $0.1^{\circ} \mathrm{s}^{-2}$ by the control electronics, resulting in, typically, $2 \mathrm{~s}$ for an azimuthal turnaround.

Elevation drift speeds of $10^{\prime \prime} \mathrm{s}^{-1}$ or elevation steps of $40^{\prime \prime}-$ $100^{\prime \prime}$ in adjacent azimuth scans provide adequate spatial sampling of the sky by the detector arrays.

Three primary scan modes are implemented (Fig. 9): "cap," a circle centered on a target right ascension/declination; "box," a rectangle in azimuth and elevation centered on a target right ascension/declination; and "quad," an arbitrary quadrilateral specified by its four corners in right ascension and declination.

\section{POINTING SENSORS}

The primary pointing sensors for BLAST are a pair of CCDbased star cameras and fiber optic rate gyroscopes. The star cameras provide absolute pointing and the gyros provide velocity information that can be integrated to allow interpolation of the gondola's attitude between star camera solutions. Coarse attitude determination is provided by several additional sensors: in elevation, there is an encoder on the elevation axis and a tilt sensor on the inner frame; in azimuth, there is a Sun sensor, a differential GPS unit, and a magnetometer. The system provides postflight pointing reconstruction to $\lesssim 5^{\prime \prime}$ rms.

\subsection{Star Cameras}

Four primary factors drive the design of the star cameras: (1) an absolute pointing accuracy of $\sim 5^{\prime \prime}$ is required to oversample the diffraction-limited size of the $250 \mu \mathrm{m}$ beam; (2) integration times (and hence efficiency) have to be short enough to avoid significant smearing at the maximum normal scan angular velocity of the gondola $\left(0.1^{\circ} \mathrm{s}^{-1}\right)$; (3) the system must always detect stars to calibrate gyroscope drift; and (4) the frequency of the solutions must be high enough to control the $1 / f$ random walk noise in the integrated gyroscopes.

To meet these requirements, we incorporated two star cameras for redundancy, and to enable increased positional accuracy in postflight processing. A detailed description of the cameras can be found in Rex et al. (2006). The cameras each use a Nikon lens with a $200 \mathrm{~mm}$ focal length and a $100 \mathrm{~mm}$ aperture to produce a $2.5^{\circ} \times 2.0^{\circ} \mathrm{FOV}$ with $7^{\prime \prime}$ pixels. Each camera is controlled by its own PC/104, $300 \mathrm{MHz}$ Celeron computer, which calculates pointing solutions for the gondola at a rate of $\sim 1.5 \mathrm{~Hz}$. The computers command the CCD cameras via FireWire, control the focus and aperture size using stepper motors via a serial port, and regulate the temperature of the camera using a small USB DAQ module. The entire system is contained in a pressure vessel to allow the operation of the hard drives, control the thermal environment, and maintain mechanical rigidity. Since the cameras operate during the day, the dominant source of noise is from the sky background, despite the altitude of the experiment. A Nikon R60 red filter is used to attenuate the background. In addition, a $1.2 \mathrm{~m}$ long cylindrical baffle is attached to the front of each camera to reduce stray-light contamination beyond $10^{\circ}$ from the optical axis.

The software controlling the camera in flight provides real-time pointing information by analyzing the star patterns in the CCD frames. The pointing algorithm locates blobs in the camera image, rejecting the known bad pixels. The best-fit positions of star 
candidates are then used by a pattern recognition algorithm to identify a unique constellation matching the observed angular separations in a star catalog (Guide Star Catalog 1.1; see Lasker et al. 1987). The magnitude limit of the catalog is chosen manually, and no brightness information for the stars is otherwise used. The algorithm is aided by an approximate pointing solution from the flight computer, required to be accurate to $\sim 5^{\circ}$ (see $\S 7.3$ ) to reduce the number of candidate star identifications. A visual magnitude limit of 9 was required to obtain sufficient completeness. A "Lost in Space" algorithm based on the Pyramid technique (Mortari et al. 2001) was also implemented to be used in case the approximate solution was found to be unreliable (which never happened during the BLAST flights).

Once the CCD object centroids are matched to stars with known coordinates $(\alpha, \delta)$, the pointing solution is calculated, parameterized by the celestial coordinates of the center pixel, $\alpha_{0}$ and $\delta_{0}$, and the roll of the camera, $r$. A model in which the image is assumed to be a perfect gnomic tangent-plane projection, with the tangent point at $\alpha_{0}$ and $\delta_{0}$, is used to project each star right ascension and declination into the plane of the CCD. The rms distance between the CCD and model star coordinates is then minimized using an iterative Newton solver with respect to the three model parameters. This procedure produces pointing solutions with uncertainties of $\sim 3.5^{\prime \prime}$ and $\sim 200^{\prime \prime}$ for the position of the tangent point and of the roll, respectively. A postflight comparison of simultaneous pointing solutions from both cameras results in an rms uncertainty of $<2^{\prime \prime}$.

\subsection{Gyroscopes}

Fiber optic rate gyroscopes (FOGs) are used to extrapolate the star camera absolute attitude to provide pointing information at each detector time sample. Two redundant sets of three FOGs are mechanically arranged to measure the gondola angular velocity along orthogonal axes. One of these sets uses ECORE 2000 analog gyroscopes, while the other uses DSP 3000 digital output gyroscopes from the same company. ${ }^{30}$ They have an angle random walk of $5^{\prime \prime} \mathrm{s}^{-1 / 2}$ and $4^{\prime \prime} \mathrm{s}^{-1 / 2}$, respectively. Since FOGs are sensitive to magnetic fields (Bohm et al. 1982), they were wrapped in a $250 \mu \mathrm{m}$ thick $\mu$-metal sheet. This reduced the signal induced from the Earth's magnetic field by a factor of $\sim 10$. The gyroscope assembly is temperature controlled to mitigate bias drifts. For BLAST05 both sets worked flawlessly. In BLAST06 two out of the three digital gyroscopes stopped working temporarily, possibly due to a cosmic-ray interaction with the gyro electronics. A power cycle of the gyroscope itself restored function. Postflight attitude reconstruction of the 2005 and 2006 data shows that the FOGs performed at near to their specified sensitivity.

\subsection{Coarse Sensors}

Two distinct Sun sensors were used on BLAST for coarse azimuth determination. For BLAST03 and BLAST05, a linear $\mathrm{CCD}$ behind a thin slit was used. It was replaced by an array of photodiodes arranged in an azimuthally oriented ring for BLAST06. The photodiode Sun sensor was smaller, lighter, and consumed less power ( $5 \mathrm{~W}$ compared to $50 \mathrm{~W}$ ) than the CCD-based sensor.

The photodiode Sun sensor is a small, all-in-one unit. A PC/104 embedded computer system is mounted below a dodecagonal ring. Twelve photodiodes are mounted radially around the ring, and the ring is mounted with its axis parallel to the azimuth axis. The intensities of light incident on the photodiode with the highest intensity and on its two nearest neighbors are compared and fitted

$30 \mathrm{KVH}$ Industries, Inc. to a $\cos \theta$ function to determine the azimuth relative to the Sun. The flight computer calculates the azimuth of the Sun to determine the azimuth of the gondola. The CCD Sun sensor, used during the first science flight, had a short-time, relative precision of $2^{\prime}$, but an overall absolute accuracy of $5^{\circ}$. The photodiode Sun sensor, used during the second science flight, had a precision of $4^{\prime}$, with an absolute accuracy of $5^{\circ}$. Both units provide azimuthal data at $>5 \mathrm{~Hz}$.

BLAST's second coarse azimuth sensor was an ADU5 differential GPS unit. ${ }^{31}$ The four required antennae were installed on booms above the Sun shields to minimize multipath reflections from the gondola (see Fig. 6). This orientation was predicted to provide better than $10^{\prime} \mathrm{rms}$ absolute pointing. However, for BLAST05, the GPS stopped providing azimuthal solutions before the instrument reached float altitude and never recovered. For BLAST06, the GPS did not provide solutions until $110 \mathrm{hr}$ after launch. It then began providing solutions and the unit operated with a precision from $0.1^{\circ}$ to $0.2^{\circ} \mathrm{rms}$. The cause of the irregular reliability of the GPS is uncertain, but may be due to a lack of thermal rigidity in the antenna mounts. The position, altitude, velocity, and time data provided by the GPS unit worked well at all times.

BLAST's third and most reliable coarse azimuth sensor was a three-axis, flux-gate magnetometer, ${ }^{32}$ which was used to determine the gondola's attitude relative to the Earth's magnetic field. The magnetic field orientation was determined using the World Magnetic Model. ${ }^{33}$ Even though BLAST passed close to the magnetic pole, the model was accurate enough and the gondola pendulations small enough that the magnetometer-based pointing solution was good to $5^{\circ}$ peak to peak in both science flights.

\section{THERMAL ENVIRONMENT}

The LDB thermal environment is characterized by continuous but variable solar illumination combined with small coupling to the atmosphere. Extensive shielding is used to regulate the temperature of the instrument. Following the design of BOOMERANG (Crill et al. 2003), BLAST is surrounded by shields comprised of $24 \mu \mathrm{m}$ thick aluminized Mylar applied to $2.5 \mathrm{~cm}$ thick polystyrene open-celled foam. The Mylar side faces out on all surfaces that may be exposed to the Sun. While bare aluminum surfaces can be expected to reach temperatures in excess of $130^{\circ} \mathrm{C}$ in the LDB float environment, Sun-facing aluminized Mylar surfaces reach temperatures of around $45^{\circ} \mathrm{C}$ due to their high ratio of thermal IR emissivity to visible light absorptivity. For telescope azimuth ranges of $\pm 60^{\circ}$ from anti-Sun, the shielding blocks direct radiation from reaching the optics and all electronics, except for the pivot motor controller and Sun sensor. The actual angles of Sun avoidance have a weak dependence on the elevation of the telescope.

The majority of the electronics dissipate their heat radiatively. A high IR emissivity is achieved by painting electronics enclosures with white paint. ${ }^{34}$ Painting the interiors of boxes white aids in radiative transfer between the electronics and the box. This is effective for all boxes, except for the detector readout electronics boxes, each of which dissipates about $100 \mathrm{~W}$. To cool these boxes, emissive vertical plates were installed between each card to couple heat from the electronics to the top and bottom walls of the boxes. A closed-cycle fluid cooling loop was used to distribute the heat to the gondola frame.

\footnotetext{
31 Magellan Navigation, Inc.

32 Applied Physics, model 534.

33 WMM-2005, National Geospatial-Intelligence Agency (NGA).

34 We used a variety of generic spray-on epoxy enamel.
} 
TABLE 4

BLAST Temperatures $\left({ }^{\circ} \mathrm{C}\right)$

\begin{tabular}{|c|c|c|c|c|c|c|c|}
\hline \multirow[b]{2}{*}{ Component } & \multicolumn{2}{|c|}{ BLAST03 } & \multicolumn{2}{|c|}{ BLAST05 } & \multicolumn{2}{|c|}{ BLAST06 } & \multirow[b]{2}{*}{ Model } \\
\hline & Trop & Diurnal & Trop & Diurnal & Trop & Diurnal & \\
\hline ACS 5V DCDC ${ }^{\mathrm{a}}$ & 25 & $51-23$ & 15 & $62-46$ & 3.6 & $41-36$ & $\ldots$ \\
\hline ACS case $^{\mathrm{b}}$ & $\ldots$ & $\ldots$ & -30 & $21-1$ & -21 & $16-10$ & $28-13$ \\
\hline PV case $^{\mathrm{c}}$ & $\ldots$ & $\ldots$ & -21 & $21-13$ & -21 & $17-12$ & $18-4$ \\
\hline 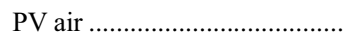 & -5 & $26-1$ & -7 & $35-24$ & -6 & $24-29$ & $\ldots$ \\
\hline Preamp case $^{\mathrm{d}}$ & -20 & 10 to -10 & $\ldots$ & $31-19$ & -5 & $25-21$ & $25-19$ \\
\hline 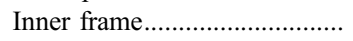 & -16 & -2 to -27 & -22 & 14 to -2 & -32 & -2 to -5 & $\ldots$ \\
\hline Batteries $^{\mathrm{e}} \ldots \ldots \ldots \ldots \ldots \ldots \ldots \ldots$ & 10 & $17-10$ & -3 & $21-10$ & -6 & $19-17$ & $16-0$ \\
\hline Solar panels ${ }^{\mathrm{f}} \ldots \ldots \ldots \ldots \ldots \ldots \ldots$ & $\ldots$ & $\ldots$ & -30 & $66-5$ & -29 & $79-58$ & 85 \\
\hline 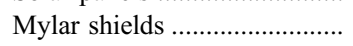 & -30 & 17 to -55 & -34 & 18 to -4 & -38 & $20-10^{\mathrm{g}}$ & $\ldots$ \\
\hline 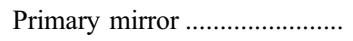 & -24 & -8 to -38 & $\ldots$ & 8 to -15 & -17 & -6 to -9 & -25 to -10 \\
\hline 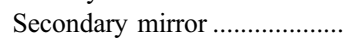 & -33 & 0 to -70 & $\ldots$ & $\ldots$ & -26 & -13 to $-16^{\mathrm{h}}$ & $\ldots$ \\
\hline 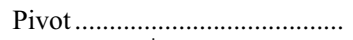 & -33 & 15 to -50 & -38 & 20 to -5 & -35 & $57-36^{\mathrm{i}}$ & $\ldots$ \\
\hline Outer frame $^{\mathrm{j}} \ldots \ldots \ldots \ldots \ldots \ldots \ldots$ & -11 & -1.4 to -37 & -30 & 5 to -13 & -24 & 2 to -3 & 25 to -5 \\
\hline 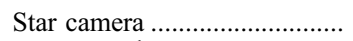 & $\ldots$ & $\ldots$ & -11 & $15-1$ & -12 & $6-1$ & $18-5$ \\
\hline Sun sensor ${ }^{\mathrm{k}} \ldots$ & 0 & 31 & -23 & $43-30$ & $\ldots$ & $\ldots$ & $\ldots$ \\
\hline
\end{tabular}

NOTES.-BLAST temperatures in the three flights, and as predicted by a Thermal Desktop (http://www.thermaldesktop.com) model for BLAST06. The model predicted larger diurnal variations than observed, partially because BLAST06 followed a very southerly track, where the Sun elevation varied less than the simulation's track. The "Trop" column gives the minimum temperature the component reached during ascent through the tropopause. The "Diurnal" column gives the daily range of temperatures. In some cases, there was slowly increasing temperature over and above the diurnal variation due to UV degradation of the Mylar shields.

${ }^{\text {a }}$ The inside of the case was painted white for BLAST06.

${ }^{\mathrm{b}}$ Partially painted white.

c Painted white.

d Painted white + cooling loop.

e Packaged in a foam box.

${ }^{\mathrm{f}}$ The model neglects current draw.

Increased by $20^{\circ} \mathrm{C}$ over 12 days.

${ }^{\mathrm{h}}$ Increased by $5^{\circ} \mathrm{C}$ over 12 days.

${ }^{\mathrm{i}}$ Increased by $4^{\circ} \mathrm{C}$ over 12 days.

jeasured at the lock motor near the elevation bearing.

${ }^{\mathrm{k}}$ With cooling pump to heat exchanger.

The thermal strategy was effective in keeping the temperatures of essentially all electronics well within their operational range. A shield added to BLAST06 to keep the Sun from illuminating the secondary mirror reflected solar radiation back onto the pivot motor controller. This increased the average temperature of the pivot by up to $20^{\circ} \mathrm{C}$ compared to what was experienced in BLAST05. As a result, two nonessential observations where shortened when the motor controller temperature exceeded $62.5^{\circ} \mathrm{C}$.

For BLAST06, the primary mirror was thermally isolated from the inner frame with G10 spacers. Approximately 12 layers of Mylar superinsulation ${ }^{35}$ were added in the gap between the mirror and the frame. This stabilized the in-flight temperature, which exhibited a diurnal excursion of about $\pm 1.5^{\circ} \mathrm{C}$.

See Table 4 and Figure 10 for a list of temperatures achieved during the various BLAST flights.

\section{POWER SYSTEM}

Solar panels provide power to the flight electronics. They are mounted at the back of the telescope on the support structure for the Sun shields (see Fig. 6). The panels face the Sun from only one side and are radiatively cooled to the sky from the other side.

During normal operation, BLAST requires $500 \mathrm{~W}$, divided between two completely isolated systems: the receiver, and the rest of the gondola (see Table 5). During LOS operations, an additional $150 \mathrm{~W}$ are required to power the transmitters. Power is pro-

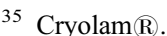

vided by the solar power system, ${ }^{36}$ which charges NiMH batteries. ${ }^{37}$ The arrays provide $1250 \mathrm{~W}$ for normal incidence at float. The arrays are thus able to provide full power to the experiment up to a Sun-array angle of $>60^{\circ}$. The batteries are essentially only used during launch and ascent.

As a NiMH battery reaches its top-off voltage, its charge efficiency decreases, leading to heating of the battery. In addition, the top-off voltage of NiMH batteries decreases with increased temperature. This combination can lead to thermal runaway if the batteries are charged with a fixed top-off voltage. For BLAST, the charge controllers were modified to allow the flight computer to reduce the top-off voltage as the battery temperature increases, permitting full battery charge without thermal runaway.

\section{FLIGHT PLANNING}

BLAST functions autonomously using schedule files that consist of a sequential list of observations or actions as a function of the local sidereal time. This system is robust against temporary system failures because the telescope only needs to know the current time and location to resume operation on recovery. Using a local sidereal clock rather than a clock fixed in some time zone, it is possible to account for purely astronomical visibility constraints (such as the right ascension of the Sun and of the astronomical targets) using a static description. However, the amount of time

36 MEER Instruments, Palomar Mountain, CA, http://www.meer.com; and SunCat Solar, Phoenix, AZ.

37 Cobasys Model 9500. 


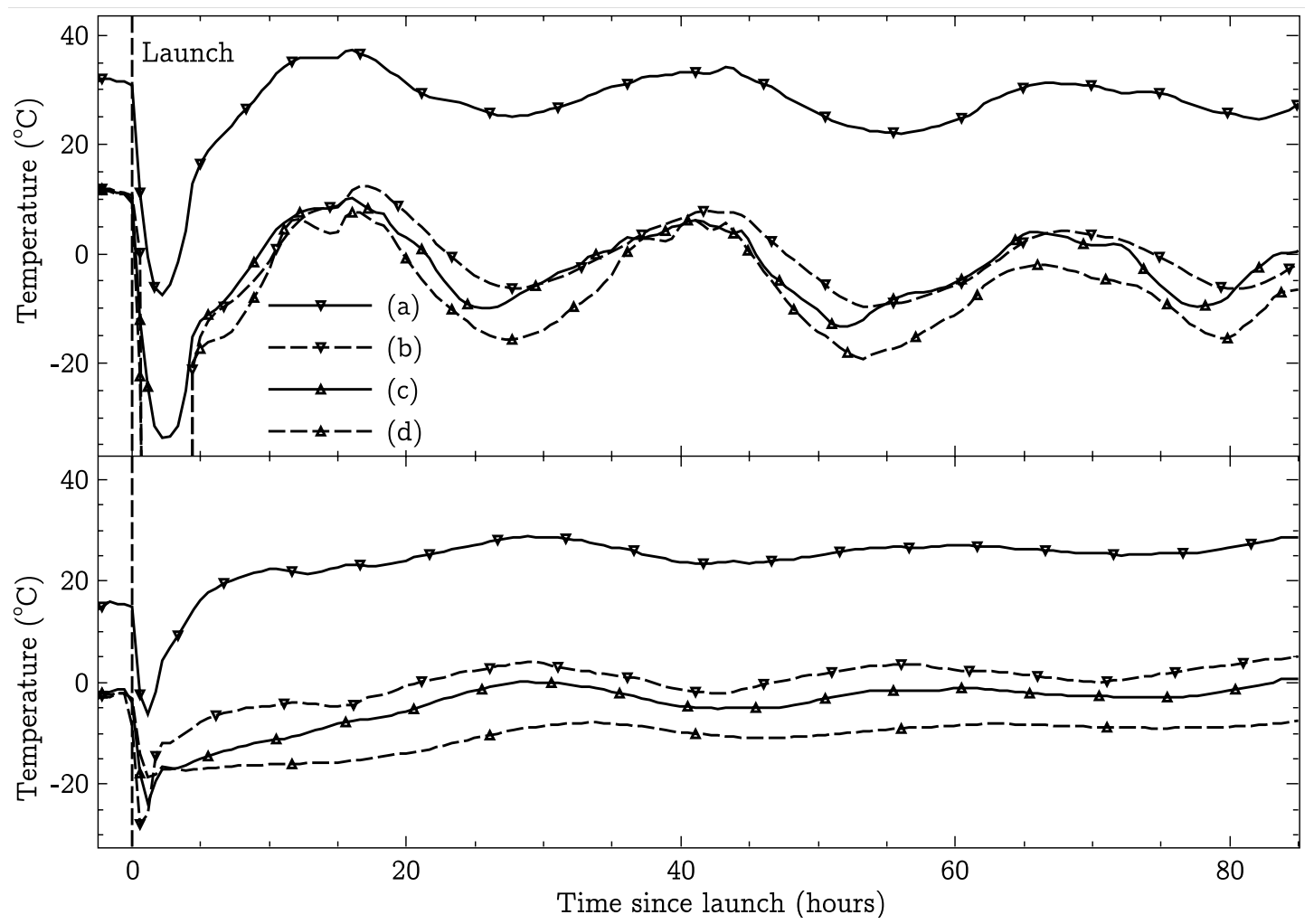

Fig. 10.-Temperatures during the 2005 (top) and 2006 (bottom) flights. Several gondola temperatures are plotted for the period starting just before launch and extending through the first few days from both science flights. Dramatic cooling in the troposphere, reheating in the stratosphere, and diurnal variations due to variation of the solar elevation angle are visible in all traces. The curves are $(a)$ the pressure vessel containing the data acquisition computers, $(b)$ the gondola inner frame, $(c)$ the elevation axis motor, and $(d)$ the primary mirror. The temperature difference between high-power consumption electronics $([a])$ and more passive components ([b] and $[c]$ ) is only slightly larger at float than it is on the ground. The primary mirror has the best geometry for cooling radiatively and therefore attains the coldest temperature. Notice the relatively large variations in the primary mirror temperature in the 2005 flight, which caused us to recognize the need for in-flight focusing capability combined with better insulation of the primary.

TABLE 5

The Steady State Power Budget for Blast

\begin{tabular}{|c|c|}
\hline Component & $\begin{array}{c}\text { Power Consumption } \\
\text { (W) }\end{array}$ \\
\hline \multicolumn{2}{|c|}{ ACS Side } \\
\hline Pointing motors ${ }^{\mathrm{a}}$.. & 85 \\
\hline Flight computers ${ }^{\mathrm{b}}$ & 55 \\
\hline 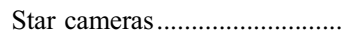 & 55 \\
\hline $\mathrm{ACS}^{\mathrm{c}}$ & 30 \\
\hline Gyro heaters ${ }^{\mathrm{d}} \ldots \ldots \ldots \ldots \ldots \ldots \ldots \ldots$ & 30 \\
\hline Auxiliary systems ……................. & 15 \\
\hline Differential GPS ............................. & 5 \\
\hline BLAST06 sun sensor................ & 5 \\
\hline 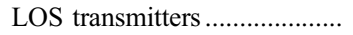 & 150 \\
\hline ACS total ................................ & 430 \\
\hline
\end{tabular}

\begin{tabular}{ll}
\hline \multicolumn{2}{c}{ DAS Side } \\
\hline DAS .................................... & 113 \\
Preamps and cryostat ……..... & 113 \\
DAS total .......................... & 225 \\
\hline
\end{tabular}

Notes.-When slewing, the pointing motors may have short-term excursions up to $560 \mathrm{~W}$, nearly doubling BLAST's power consumption. The LOS transmitters are only on at the start of the flight and do not contribute to the steady state budget.

a Steady state.

${ }^{\mathrm{b}}$ Includes everything else in the pressure vessel.

c ACS crate only.

d Mean value. the telescope spends on a given observation varies depending on telescope longitudinal drifts.

Flight schedules are calculated on the ground, in advance of the launch using best estimates for the launch time, flight path, and length. Given the uncertainties in these flight parameters and the complexity of the observations (many small maps scattered across the sky, of which only a subset are available at a given instant), a schedule file generator was developed to automate the process. This system interprets a high-level description of the desired scientific observations, such as "map a circular region centered at a given RA and DEC with a given radius." It then accounts for a number of parameters, such as the launch date and time, projected duration of flight, the start longitude and latitude, a guess for the termination longitude, a latitude range over which the payload may drift, and the position of the Sun and the Moon. With this information, the scheduler generates an optimized list of consecutive actions. At each instant, observations that are possible under the current visibility constraints are scheduled in order of scientific priority. At regular intervals, calibration observations are assigned maximum priority, so that sensitivity, beam shape, and pointing variations may be tracked throughout the flight. Once a schedule file is generated, a simple model for the power usage of the system and the charge rate of the solar panels as a function of Sun incidence angle is used to calculate the available battery charge over time. The schedule is modified by hand if this model indicates that the batteries will have less than $50 \%$ of the total charge capacity at any point in the flight. Schedules are then tested using a simulator that mimics the scanning motion of the telescope to determine the coverage (effective integration time 

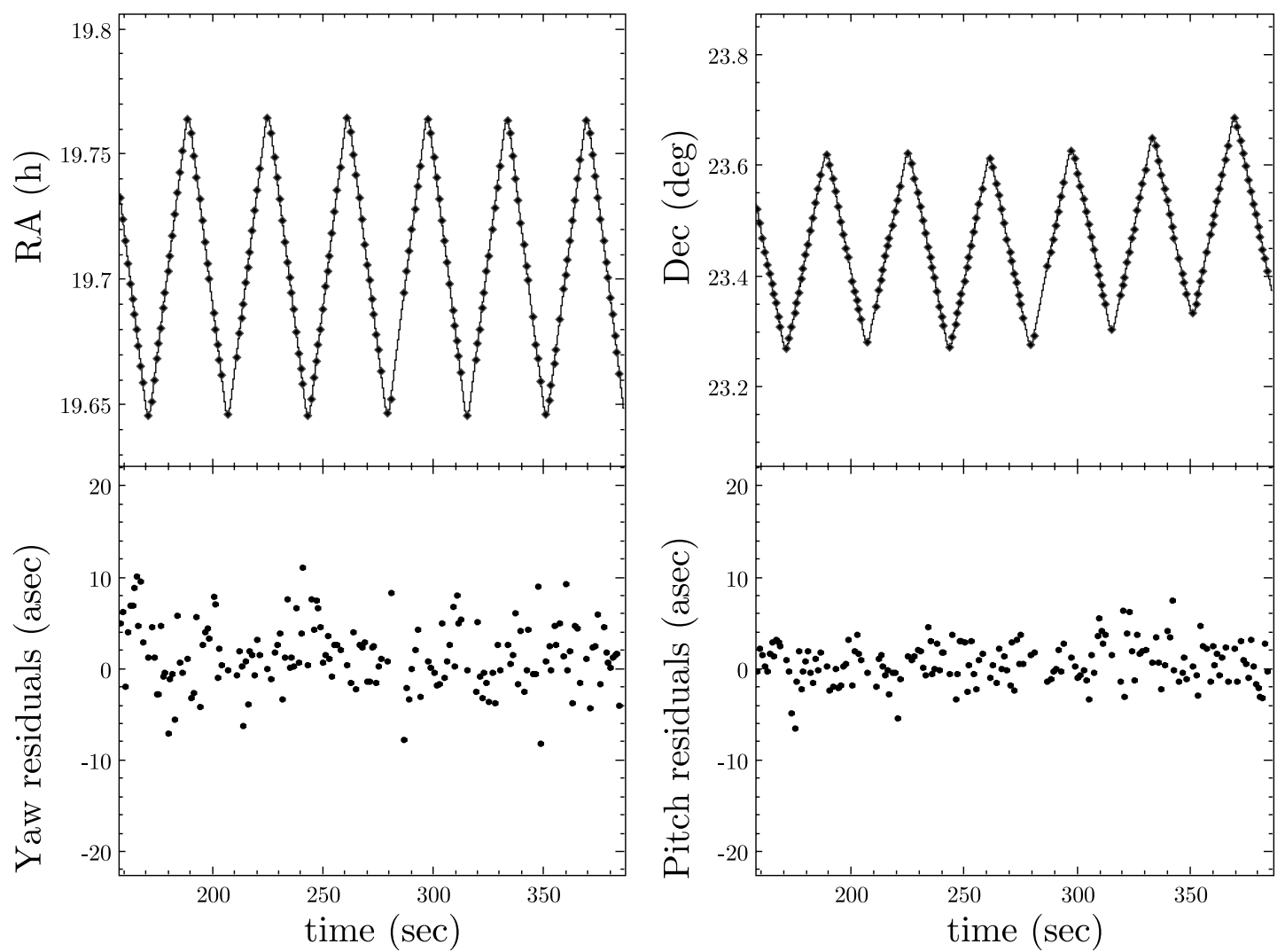

FIG. 11.-Example of pointing reconstruction from the BLAST05 data. In the top panels, the solid lines represent the reconstructed pointing solution obtained by integrating one set of gyroscopes onto one of the two star cameras. Filled circles represent the positions reported by the other star camera, which is not used in the solution. The bottom panels show the residuals as yaw $[\sim \Delta$ R.A. cos (decl.)] and pitch (coincident with declination in this particular case). The yaw residuals are slightly wider, since the telescope scans in azimuth, which is close to right ascension at the BLAST05 latitudes. The projection of the gondola angular velocity along the yaw axis is always larger than the pitch axis for the elevation range of the telescope $\left(25^{\circ}-60^{\circ}\right)$, and hence the star centroids in star camera images have larger measurement errors in that direction The overall error of the pointing solution can be obtained by summing in quadrature the standard deviations of the residuals, yielding $\simeq 4.2^{\prime \prime}$ rms. Improvements to this result are discussed in $\S 11$.

across the maps) and cross linking among each of the maps for a given flight trajectory.

Six schedule files are generated for each launch opportunity. The latitude of the gondola can change by as much as $\sim 15^{\circ}$ during a flight, hence three different schedules are created in latitude bands that are $6^{\circ}$ wide, with $1^{\circ}$ of overlap. The gondola uses the time and payload position reported by the GPS to decide which schedule file to use. Two sets of these three schedules are made: the first set assumes that the instrument is working with the target sensitivities; the second assumes degradations of the telescope beam size by a factor of $\sqrt{2}$ and sensitivity by a factor of 2 . At the beginning of the flight, the sensitivities are estimated from scans across calibrators. Based on this information, the ground station team can decide which of the two sets of schedule files the instrument should use and can switch between the two using a single command.

\section{POINTING RECONSTRUCTION}

Postflight pointing reconstruction estimates the rotation (attitude) of the gondola with respect to the celestial sphere as a function of time, providing right ascension, declination, and rotation angle information at each detector sample. The star camera provides absolute attitude on an unevenly sampled time grid $(\sim 1.5 \mathrm{~s})$, with an accuracy of $<2^{\prime \prime} \mathrm{rms}$. Each solution is sampled at a known phase with respect to the detectors. The detector and gyroscope sampling are synchronized; therefore, the integration of the gyroscopes gives one estimate of the gondola attitude. The star camera is used to correct the random walk drift induced by the integrated gyroscope noise and to give an estimate of the integration constant. BLAST implements a Kalman filter approach to estimate the attitude, expressed as the state quaternion $q(t)$, a four-dimensional quantity describing the solid body rotation of the gondola. The nonlinear state model representing the gondola is defined as

$$
\begin{gathered}
\boldsymbol{b}_{n+1}=\boldsymbol{b}_{n}+\boldsymbol{w}_{b_{n}}, \\
q_{n+1}=\left[\begin{array}{c}
1 \\
\frac{1}{2}\left(\boldsymbol{\omega}_{n}+\boldsymbol{b}_{n}+\boldsymbol{w}_{\omega_{n}}\right) \Delta T
\end{array}\right] q_{n},
\end{gathered}
$$

where $\boldsymbol{\omega}_{n}$ is the gyro angular velocity with $\boldsymbol{w}_{\omega_{n}}$ its noise, $\boldsymbol{b}_{n}$ is the gyro bias, $\boldsymbol{w}_{b_{n}}$ is the filter process noise, and $\Delta T$ is the time resolution of the system $(10 \mathrm{~ms})$. The filter is run forward and backward in time and the two solutions are weighted together, using the Kalman covariance matrix output from the filter as the weight. Using just one star camera and the digital gyros, the final averaged attitude is better than $5^{\prime \prime} \mathrm{rms}$; one example is shown in Figure 11 using data from the BLAST05 flight. The achieved precision is sufficient for BLAST beam sizes but can be improved once the second set of gyroscopes and the additional star camera are included in the solution. An improvement of $\sqrt{2}$ to 2 better can be reasonably expected. The pointing solution is referenced to the star camera reference frame and needs to be rotated into the submillimeter array coordinate frame. Bright optical and submillimeter point sources are used to evaluate the rotation quaternion 
$Q$. This correction is ideally static but, in practice, was found to be a weak function of time. As shown in Figure 6, the star cameras are mounted at the top of the inner frame. A structural deformation of the frame can modify the relative orientation between the cameras and the submillimeter beam. Therefore, several pointing calibrators are observed each time the geometry of the scan changes. This effect can be as large as $35^{\prime \prime}$ and has an $80 \%$ correlation with the elevation and a $25 \%$ correlation with the temperature of the inner frame. Although it is possible to model the effect based on the gondola thermometry and attitude, $Q$ is such a weak function of time that this was found unnecessary.

\section{CONCLUSIONS}

BLAST is an instrument designed to probe the local and high- $z$ universe at short submillimeter wavelengths. Over the course of two science flights, BLAST has addressed a broad range of Galactic and extragalactic topics. The balloon environment requires a novel combination of ground and satellite technology and techniques. This paper is intended to serve as a reference for a suite of science papers from the BLAST05 and BLAST06 flights.
The BLAST collaboration acknowledges the support of NASA through grants NAG5-12785, NAG5-13301, and NNGO-6GI11G, the Canadian Space Agency (CSA), the Science and Technology Facilities Council (STFC), Canada's Natural Sciences and Engineering Research Council (NSERC), the Canada Foundation for Innovation, the Ontario Innovation Trust, the Puerto Rico Space Grant Consortium, the Fondo Istitucional para la Investigacion of the University of Puerto Rico, and the National Science Foundation Office of Polar Programs; C. B. N. also acknowledges support from the Canadian Institute for Advanced Research. L. O. would like to acknowledge Pietro Bolli for his help with physical optics simulations during the testing phase of the BLAST06 telescope. We would also like to thank the Columbia Scientific Balloon Facility (CSBF) staff for their outstanding work, the Precision Machining Group at Lawrence Livermore Laboratory, the support received from Empire Dynamic Structures in the design and construction of the gondola, Daniele Mortari for helpful discussions in the development of the Pyramid code, Dan Swetz for building the Fourier transform spectrometer, and Luke Bruneaux, Kyle Lepage, Danica Marsden, Vjera Miovic, and James Watt for their contribution to the project.

\section{REFERENCES}

Ade, P. A. R., Pisano, G., Tucker, C., \& Weaver, S. 2006, Proc. SPIE, 6275, 26

Bock, J. J., Delcastillo, H. M., Turner, A. D., Beeman, J. W., Lange, A. E., \& Mauskopf, P. D. 1996, in Submillimetre and Far-Infrared Space Instrumentation, ed. E. J. Rolfe \& G. Pilbratt (ESA SP-388; Noordwijk: ESA), 119

Bock, J. J., Glenn, J., Grannan, S. M., Irwin, K. D., Lange, A. E., Leduc, H. G., \& Turner, A. D. 1998, Proc. SPIE, 3357, 297

Bohm, K., Petermann, K., \& Weidel, E. 1982, Opt. Lett., 7, 180

Chapin, E. L., et al. 2008, ApJ, 681, 428

Chattopadhyay, G., Glenn, J., Bock, J. J., Rownd, B. K., Caldwell, M., \& Griffin, M. J. 2003, IEEE Trans. Micro. T. Tech., 51, 2139

Crill, B. P., et al. 2003, ApJS, 148, 527

Devlin, M. J., et al. 2004, Proc. SPIE, 5498, 42

Griffin, M. J., Bock, J. J., \& Gear, W. K. 2002, Appl. Opt., 41, 6543

Griffin, M. J., Swinyard, B. M., \& Vigroux, L. G. 2003, Proc. SPIE, 4850, 686

Hargrave, P., Waskett, T., Lim, T., \& Swinyard, B. 2006, Proc. SPIE, 6275, 36

Holland, W., et al. 2006, Proc. SPIE, 6275, 45

Hughes, D. H., et al. 2002, MNRAS, 335, 871

Lamarre, J. M., et al. 1998, ApJ, 507, L5

Lasker, B. M., Jenkner, H., \& Russell, J. L. 1987, NASA STI/Recon Tech. Rep. N, 88,30547

Mather, J. C. 1984, Appl. Opt., 23, 584

Mortari, D., Junkins, J. L., \& Samaan, M. A. 2001, Lost-in-Space Pyramid Algorithm for Robust Star Pattern Recognition, AAS Paper 01-004 of the 22th Annual AAS Rocky Mountain Guidance and Control

Olmi, L. 2001, Int. J. IR Millimeter Waves, 22, 791

2002, Proc. SPIE, 4849, 245

Olmi, L., \& Bolli, P. 2007, Appl. Optics, 46, 4092

Patanchon, G., et al. 2008, ApJ, 681, 708

Rex, M., Chapin, E., Devlin, M. J., Gundersen, J., Klein, J., Pascale, E., \& Wiebe, D. 2006, Proc. SPIE, 6269, 109

Rownd, B., Bock, J. J., Chattopadhyay, G., Glenn, J., \& Griffin, M. J. 2003, Proc. SPIE, 4855, 510

Truch, M. D. P. 2007, Ph.D. thesis, Brown Univ.

Truch, M. D. P., et al. 2008, ApJ, 681, 415

Turner, A. D., et al. 2001, Appl. Opt., 40, 4921 